\title{
Two-Sided Allocation Problems, Decomposability, and the Impossibility of Efficient Trade*
}

\author{
David Delacrétaz ${ }^{\dagger} \quad$ Simon Loertscher Leslie M. Marx $^{\ddagger} \quad$ Tom Wilkening ${ }^{\Uparrow}$
}

October 30, 2018

\begin{abstract}
Previous literature has shown that private information is a transaction cost that prevents efficient reallocation in two-sided setups with bilateral trade or homogeneous goods. We derive conditions under which the impossibility of efficient trade extends to rich environments in which buyers and sellers have multi-dimensional private types, accommodating many-to-many trades and heterogeneous objects. If agents can be decomposed into unit constituents, the allocation problem can be represented as an assignment game and impossibility obtains through a generalization of Shapley's (1962) result that buyers and sellers are complements. We introduce a general family of payoff functions that ensures decomposability and thus impossibility.
\end{abstract}

Keywords: mechanism design, assignment games, impossibility theorems, decomposability, sizedependent discounts JEL Classifications: C72, D44, D61

*We thank two anonymous reviewers and the lead editor (Alessandro Pavan) for comments and suggestions that have helped us to improve the exposition of the paper. We are also grateful to Juan Carlos Carbajal, John Hatfield, John Ledyard, Claudio Mezzetti, Ellen Muir, Mike Ostrovsky, Larry Samuelson, Alex Teytelboym, M. Utku Ünver, Steve Williams, Jun Xiao, M. Bumin Yenmez, and seminar audiences at the University of Melbourne, the University of Technology Sydney, the University of Queensland, the Econometric Society European Meeting 2014, the Econometric Society Australasia Meeting 2016, the European Economic Association Meeting 2016, and the Australasian Economic Theory Workshop 2018 for their valuable comments. Financial support through an Eminent Research Scholar grant from the Faculty of Business and Economics at the University of Melbourne, the Australian Research Council grant DP160101350, the Centre for Market Design, and the Samuel and June Hordern Endowment is also gratefully acknowledged.

${ }^{\dagger}$ Nuffield College, University of Oxford, New Road, Oxford, OX1 1NF, United Kingdom. Email: david.delacretaz@nuffield.ox.ac.uk.

${ }^{\ddagger}$ Department of Economics, Level 4, FBE Building, University of Melbourne, 111 Barry St, Victoria 3010, Australia. Email: simonl@unimelb.edu.au.

${ }^{\S}$ The Fuqua School of Business, Duke University, 100 Fuqua Drive, Durham, NC 27708, USA: Email: marx@duke.edu.

IDepartment of Economics, Level 3, FBE Building, University of Melbourne, 111 Barry St, Victoria 3010, Australia. Email: tom.wilkening@unimelb.edu.au. 


\section{Introduction}

The Coase Theorem provides the essential insight that any efficiency rationale for government intervention must be based on transaction costs. Absent such costs, the allocation that results from mutually beneficial trade will be efficient irrespective of initial ownership.

The mechanism design literature has shown that private information may constitute such a transaction cost. In particular, the path-breaking work by Vickrey (1961) and Myerson and Satterthwaite (1983) provides conditions under which efficient trade between privately informed buyers and sellers is impossible without running a deficit. While subsequent work by Gresik and Satterthwaite (1989), McAfee (1992), and Williams (1999) has expanded our understanding of these conditions, the literature has largely focused on homogeneous goods with single-unit demands and supplies. ${ }^{1}$ Given the role that the Coase Theorem plays in economics, understanding the conditions under which secondary markets cannot be expected to operate efficiently is of fundamental relevance.

In this paper, we derive conditions under which the impossibility of efficient trade extends to rich environments in which buyers and sellers demand and supply packages of heterogeneous objects and in which both buyers and sellers can trade with multiple agents on the other side of the market. In line with the strand of literature on the impossibility of efficient trade mentioned above, we assume private values throughout the paper. However, in contrast to most of this literature, we allow for multi-dimensional types. ${ }^{2}$

Our approach is both general and intuitive. We begin by synthesizing the literature and by showing a surprising connection between the known impossibility theorems and a result due to Shapley (1962). Shapley's result implies that when trading is one-to-one, any buyer and any seller are complements under surplus maximization - the buyer's marginal product is larger when the seller is present than when he is not and, analogously, the seller's marginal product is larger when the buyer is present than when he is not. This complementarity turns out to have important consequences when the values of buyers and the costs of sellers are private information and there exist least efficient types who should never trade: a key insight from the Vickrey-Clarke-Groves (VCG) mechanism and the literature on dominant strategy implementation is that truthful reve-

\footnotetext{
${ }^{1}$ Two exceptions are Vickrey (1961), who allows for many traders with multi-unit demands and multi-unit supplies of a homogeneous good, and Hurwicz (1972), who addresses the problem of implementing a Pareto efficient, individually rational allocation in dominant strategies in a general equilibrium model without quasi-linear payoffs.

${ }^{2}$ It is well known that with interdependent values and multi-dimensional types, efficient, incentive compatible allocations may be impossible, with or without running deficit; see, for example, Jehiel and Moldovanu (2001).
} 
lation of private information is possible if and only if each agent receives his marginal product as a transfer (plus a constant). ${ }^{3}$ Because the market maker can extract only the social gains from trade associated with a reallocation, but must pay each agent his marginal product to reveal his information, efficient trade is impossible without running a deficit when the sum of the marginal products exceeds social gains from trade. ${ }^{4}$ Shapley's result thus implies the impossibility of efficient trade for any two-sided allocation problem with one-to-one trading because the complementarity between buyers and sellers implies that the sum of marginal products always exceeds the social gains from trade. ${ }^{5}$

Building on this insight, we extend Shapley's result to develop a new impossibility theorem that applies to environments in which buyers can buy and sellers can produce multiple objects and in which buyers and sellers can trade with multiple agents on the other side of the market. We say that a buyer is decomposable if his utility function is an assignment valuation, as defined by Ostrovsky and Paes Leme (2015), and extend the concept to sellers. ${ }^{6}$ The term "decomposable" refers to the fact that such an agent can be decomposed into unit constituents who can be assigned at most one object. Obviously, this is possible if an agent's payoff function is additive in the stand-alone values associated with each object. However, decomposability is substantially more general than that and includes, among other setups, the seemingly unrelated homogeneous goods model with decreasing marginal values and increasing marginal costs.

We show that a two-sided allocation problem is assignment representable - that is, it can be represented as an assignment game between unit constituents and objects - if and only if every agent is decomposable. We then extend the results of Shapley (1962) to show that efficient trade is impossible in any assignment-representable problem.

We also introduce a new family of payoff functions satisfying what we call size-dependent discounts (SDD): Under SDD, an agent's valuation for a package of objects consists of the sum of the stand-alone utilities of these objects minus a discount that depends on the size of the package

\footnotetext{
${ }^{3} \mathrm{As}$ is well known, the VCG mechanism is an efficient mechanism that endows agents with dominant strategies. It is due to the independent contributions of Vickrey (1961), Clarke (1971), and Groves (1973). The richness of the environment in Groves (1973) does not permit dominant strategies, but the mechanism developed there endows agents with such strategies in simpler environments such as the ones studied in Groves and Loeb (1975) or here.

${ }^{4}$ We follow the literature in referring to "allocation problems" even though, in a sense, these are "reallocation problems."

${ }^{5}$ Loertscher et al. (2015) invoke Shapley's (1962) theorem to prove the impossibility of trade in allocation problems with one-to-one trading and homogeneous goods. Among other things, we extend this to all environments with oneto-one trading, without any restriction on the nature of the objects that are traded.

${ }^{6}$ Our concept of decomposition can be extended to a setting such as the one studied by Yenmez (2015), where each agent is endowed with a package of objects and can sell some of them, buy other objects, or both.
} 
but not on the objects. We show that SDD is sufficient for decomposability. ${ }^{7}$ This family includes all payoff functions with unit demand and unit supply, the homogeneous goods model with multiunit traders, additive payoffs, a version of Ausubel's (2006) heterogeneous commodities model with additively separable payoff functions, and any problems involving a mixture of agents with payoff functions of these forms.

Viewed from this angle, our results thus provide the unifying and, to our knowledge, novel insight that the underlying force behind the impossibility of efficient trade in the two-sided allocation problems of Vickrey (1961), Myerson and Satterthwaite (1983), Gresik and Satterthwaite (1989), and McAfee (1992) is that the payoff functions satisfy SDD. Of course, our results also imply that efficient trade with privately informed agents is impossible in the assignment game of Shapley and Shubik (1972), which is popular in the literature on matching with transfers, but has received relatively little attention in the mechanism design literature.

By providing a general and intuitive impossibility theorem for two-sided allocation problems under conditions that are easy to check because they relate to individual agents' payoff functions, our paper highlights the transaction cost that results from agents' strategic use of their private information about values and costs in two-sided settings. Beyond their implications for economics, along the lines discussed in the opening paragraphs, these results also emphasize a general tension between revenue and efficiency faced by market designers in two-sided settings. Moreover, SDD payoff functions are flexible and encompass as special cases payoff functions that are widely used in the literature. Nonetheless, they impose limited burden for agents' information acquisition, and for communication and computation: the only pieces of information required from an agent are his stand-alone utilities for each object and discounts for each possible package size. This contrasts with allocation problems of heterogeneous objects, for which, absent additional structure, the number of valuations required from every agent is equal to the power set of packages. ${ }^{8}$ This makes the SDD family of potential interest and value beyond the environments and problems we study in this paper.

The literature on Bayesian mechanism design has predominantly focused on settings with onedimensional types, with little attention to the connection between allocation problems and assignment games. An important implication of our approach is that a broad class of models are

\footnotetext{
${ }^{7}$ To the best of our knowledge, none of the existing definitions of substitutability that have been used in the literature is sufficient to ensure decomposability in general (see, e.g., Hatfield et al. (2012) or Hatfield et al. (2017)).

${ }^{8}$ Put differently, with $K$ objects the dimensionality of the information an agent is required to have is of order $2^{K}$ without additional structure and of order $2 K$ with SDD.
} 
assignment representable, including the two-sided market models of Vickrey (1961), Shapley and Shubik (1972), Myerson and Satterthwaite (1983), Gresik and Satterthwaite (1989), McAfee (1992), and a two-sided, additively separable version of Ausubel's (2006) model with heterogeneous commodities. Rather than being disjoint and independent problems, we demonstrate and characterize a connection among these models. ${ }^{9}$

In a recent paper, Segal and Whinston (2016) derive impossibility results that revolve around tests for a multi-valued marginal core in allocation games with monetary transfers. Yenmez (2015) provides necessary and sufficient conditions for the converse of the marginal core condition to hold. The marginal core condition and its converse are useful in providing general conditions under which the (im)possibility result holds but may be difficult to apply in practice. Our results regarding assignment-representable problems provide a complementary approach for testing for the impossibility of efficient trade. By generalizing the assignment model to accommodate package demand and supply, we derive conditions based on the primitives of the model, namely the payoff functions.

Many matching problems are also assignment representable and thus our paper connects to the literature on matching with transfers. Initiated by Koopmans and Beckmann (1957), Shapley (1962), and Shapley and Shubik (1972), with recent contributions by, among others, Bikhchandani and Ostroy (2002), Echenique et al. (2013), Chambers and Echenique (2015), and Choo (2015), the literature on matching with transfers has, beyond concerns of stability and its relation to the core, paid limited attention to individuals' incentives to reveal what is plausibly their private information. We show that it is impossible to elicit such information without running a deficit if there are least efficient types on both sides of the market that never trade. ${ }^{10}$ Yenmez (2013) studies incentive-compatible matching with transfers and proves the possibility of efficiency under the converse assumption that all types of all agents always trade.

\footnotetext{
${ }^{9}$ In a recent paper that takes a bargaining (or robust mechanism design) approach to the problem of bilateral trade with many items, Jackson et al. (2015) show that whether efficiency is possible depends on whether the two agents bargain over all items at once or independently. The difference between the possibility results that Jackson et al. (2015) obtain and the impossibility results derived here stems from a difference regarding the following assumption: in line with Myerson and Satterthwaite (1983), we assume that for every type realization of the other agent(s), an agent may be of such an unproductive type that it is efficient that he does not trade at all, while the possibility results of Jackson et al. (2015) are obtained under the assumption that the bilateral surplus over all items is positive for all type realizations for which the density is positive.

${ }^{10}$ As Shapley and Shubik (1972) show, the core of an assignment game is always nonempty. Furthermore, core payoffs are efficient. This extends to assignment-representable environments because each agent is a coalition of unit constituents in the corresponding assignment game. However, we show that no dominant strategy mechanism exists that achieves efficiency without running a deficit. Thus, we identify environments in which core payoffs exist but in which it is not possible to elicit the information required to achieve them.
} 
From a modeling perspective, our paper extends the package assignment model of Bikhchandani and Ostroy (2002) by introducing heterogeneity on the sellers' side. ${ }^{11}$ Our impossibility result implies that in one-to-one matching markets with transfers, no budget-balanced mechanism exists that is strategy-proof when least efficient types exist that should always remain unmatched. Although the settings differ, this result parallels Roth's (1982) finding that in a marriage market, in which there are no transfers, no mechanism exists that is strategy-proof for both sides and generates a stable matching.

Our paper also relates to the literature on the micro-foundations of the canonical model of price formation in markets. As first noticed by Arrow (1959), the Walrasian model is silent about the institutions that simultaneously discover and set the market clearing prices. Recent contributions by Satterthwaite et al. $(2014,2015)$ have focused on the performance of the $k$-double-auction in environments with unit traders, allowing for the possibility of correlated types and interdependent values. Our work is complementary to this research agenda. We do not restrict the mechanism that the market maker employs, other than imposing incentive and individual rationality constraints, and we allow for a general trading environment apart from imposing private values.

The remainder of this paper is organized as follows. Section 2 describes the setup. In Section 3 , we synthesize existing results to derive an impossibility result when it is efficient for all trades to be one-to-one. Section 4 contains our new result that extends the impossibility result to setups with many-to-many trading, provided that the allocation problems are assignment representable. In Section 5, we characterize assignment-representable problems and show that an allocation problem is assignment representable if all agents' payoff functions exhibit size-dependent discounts. Section 6 concludes. Proofs are in the Appendix.

\section{Setup}

A two-sided allocation problem consists of $B$ buyers $b \in \mathbb{B}, S$ sellers $s \in \mathbb{S}$, and $O$ objects $o \in \mathbb{O}$. Each buyer $b$ can consume any package $x_{b} \subseteq \mathbb{O}$. Let $\mathbb{P}:=\mathcal{P}(\mathbb{O})$ be the set of all possible packages that can be consumed and denote by $P:=2^{O}$ the cardinality of the set. ${ }^{12}$

Let $\left\{\mathbb{O}_{s}\right\}_{s \in \mathbb{S}}$ be a partition of $\mathbb{O}$. Each seller has $O_{s}$ objects $o_{s} \in \mathbb{O}_{s}$ and can produce any

\footnotetext{
${ }^{11}$ Among other things, our paper generalizes the conditions under which buyers are known to be substitutes, which is a property that is assumed to hold in parts of the analysis of Bikhchandani and Ostroy (2002).

${ }^{12}$ For instance, if there are two objects $a$ and $b$, the set of possible packages contains $\{\varnothing\},\{a\},\{b\}$, and $\{a, b\}$. The cardinality of the set is $2^{2}=4$.
} 
package $x_{s} \subseteq \mathbb{O}_{s}$. This implies that every object $o \in O$ can be produced by exactly one seller. ${ }^{13}$ We call $\mathbb{O}_{s}$ the potential set of seller $s$ and denote by $\mathbb{P}_{s}:=\mathcal{P}\left(\mathbb{O}_{s}\right)$ the set of all possible packages $s$ can produce, whose cardinality we denote by $P_{s}:=2^{O_{s}}$.

An allocation $x=\left(\left(x_{b}\right)_{b \in \mathbb{B}},\left(x_{s}\right)_{s \in \mathbb{S}}\right)$ corresponds to trades between buyers and sellers, where $x_{b}$ is the package $b$ receives and $x_{s}$ is the package $s$ produces. ${ }^{14}$ An allocation is feasible if (i) each seller produces a subset of his potential set: $x_{s} \subseteq \mathbb{O}_{s}$ for all $s \in \mathbb{S}$, (ii) each object that is consumed has been produced: $x_{b} \subseteq \cup_{s \in \mathbb{S}} x_{s}$ for all $b \in \mathbb{B}$, and (iii) each object is consumed at most once: $x_{b} \cap x_{b^{\prime}}=\varnothing$ for any $b, b^{\prime} \in \mathbb{B}$ with $b \neq b^{\prime}$. Let $X$ denote the set of feasible allocations.

Throughout, we use the term agent to refer to an individual that may be either a buyer or a seller. We assume that types are agents' own private information and are defined over the packages that they produce or consume. This implies that a buyer $b$ 's type $\mathbf{v}_{b} \in \mathbb{R}^{P}$ is a $P$-dimensional vector of values corresponding to each possible consumption package. A seller $s$ 's type $\mathbf{c}_{s} \in \mathbb{R}^{P_{s}}$ is a $P_{s}$-dimensional vector of costs corresponding to each possible production package. Let $V_{b}$ denote the set of possible types for buyer $b$ and $C_{s}$ denote the set of possible types for seller $s$. The sets $V:=\Pi_{b \in \mathbb{B}} V_{b}$ and $C:=\Pi_{s \in \mathbb{S}} C_{s}$ are the products of the sets of types, with typical elements $\mathbf{v}$ and c. We assume that $V_{b}$ and $C_{s}$ are closed and convex sets. Denote by $\mathbf{v}_{-b}$ the vector of types of buyers other than $b$ and denote by $\left(\hat{\mathbf{v}}_{b}, \mathbf{v}_{-b}\right)$ the vector $\mathbf{v}$ with the type of buyer $b$ replaced by $\hat{\mathbf{v}}_{b}$. We analogously define $\mathbf{c}_{-s}$ and $\left(\hat{\mathbf{c}}_{s}, \mathbf{c}_{-s}\right)$ for sellers.

Buyer $b$ 's total payoff takes the quasi-linear form $u_{b}\left(x_{b}, v_{b}\right)-t_{b}$, where $u_{b}$ is buyer $b$ 's utility function, $x_{b}$ is the package the buyer receives, $v_{b}$ is his type, and $t_{b}$ is a monetary transfer made by buyer $b$ to the mechanism. ${ }^{15}$ Similarly, seller $s$ 's total payoff is $t_{s}-k_{s}\left(x_{s}, c_{s}\right)$, where $k_{s}$ is seller $s$ 's cost function and $t_{s}$ is a monetary transfer made by the mechanism to seller $s$. When referring collectively to buyers' utility functions and sellers' cost functions, we refer to them as agents' payoff functions.

We normalize the utility and cost of non-trading agents to zero, i.e., $u_{b}(\varnothing, \cdot)=0$ and $k_{s}(\varnothing, \cdot)=$ 0 . We also assume that $u_{b}\left(x_{b}, \mathbf{v}_{b}\right)$ and $k_{s}\left(x_{s}, \mathbf{c}_{s}\right)$ only depend on the agents' own allocations and on their own private types. We allow for free-disposal and, without loss of generality, impose

\footnotetext{
${ }^{13}$ Here, seller $s$ producing a package $x_{s}$ can equivalently mean physical production of the objects in $x_{s}$ or figurately the sale of these objects when the seller is endowed with them.

${ }^{14}$ It may seem more accurate to think of $x$ as a reallocation of objects, in particular when each seller $s$ is initially endowed with a set of objects $\mathbb{O}_{s}$. However, the use of the terms "allocation" and "allocate" simplifies language without too severe a risk of creating confusion.

${ }^{15}$ While standard, the assumption of quasi-linear total payoffs is restrictive. As pointed out in concurrent work by Garratt and Pycia (2016), efficient bilateral trade may be possible if one relaxes this assumption.
} 
monotonicity on the utility and cost functions, that is, for all buyers, $u_{b}\left(x_{b}^{\prime}, \mathbf{v}_{b}\right) \leq u_{b}\left(x_{b}, \mathbf{v}_{b}\right)$ for any $x_{b}^{\prime} \subseteq x_{b} \subseteq \mathbb{O}$ and for all sellers, $k_{s}\left(x_{s}^{\prime}, \mathbf{c}_{s}\right) \leq k_{s}\left(x_{s}, \mathbf{c}_{s}\right)$ for any $x_{s}^{\prime} \subseteq x_{s} \subseteq \mathbb{O}_{s} \cdot{ }^{16}$

For a feasible allocation $x \in X$ and types $(\mathbf{v}, \mathbf{c})$, define the social gains from trade as

$$
W(x, \mathbf{v}, \mathbf{c}):=\sum_{b \in \mathbb{B}} u_{b}\left(x_{b}, \mathbf{v}_{b}\right)-\sum_{s \in \mathbb{S}} k_{s}\left(x_{s}, \mathbf{c}_{s}\right) .
$$

An allocation is efficient if it maximizes social gains from trade. Formally, the set of efficient allocations is

$$
X^{*}(\mathbf{v}, \mathbf{c}):=\underset{x \in X}{\arg \max } W(x, \mathbf{v}, \mathbf{c}) .
$$

Let $x^{*}(\mathbf{v}, \mathbf{c}) \in X^{*}(\mathbf{v}, \mathbf{c})$ be an efficient allocation. ${ }^{17}$ Further denote the maximum social gains from trade by $W^{*}(\mathbf{v}, \mathbf{c}):=W\left(x^{*}(\mathbf{v}, \mathbf{c}), \mathbf{v}, \mathbf{c}\right)$.

It is also useful to denote social gains from trade when a subset of buyers $\mathbb{I} \subseteq \mathbb{B}$ and a subset of sellers $\mathbb{J} \subseteq \mathbb{S}$ are excluded. For any feasible allocation $x$ of that allocation problem, the social gains from trade are

$$
W_{-\mathbb{I},-\mathbb{I}}(x, \mathbf{v}, \mathbf{c}):=\sum_{b \in \mathbb{B} \backslash \mathbb{I}} u_{b}\left(x_{b}, \mathbf{v}_{b}\right)-\sum_{s \in \mathbb{S} \backslash \mathbb{I}} k_{s}\left(x_{s}, \mathbf{c}_{s}\right) .
$$

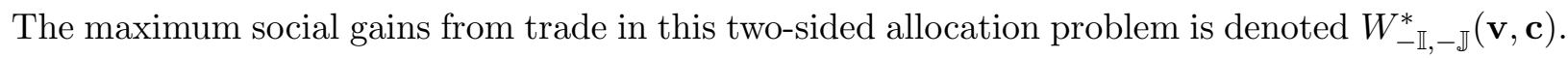
We drop dependencies on $(\mathbf{v}, \mathbf{c})$ when there is no risk of confusion.

For notational convenience, let $W_{-b, .}^{*}:=W_{-\{b\},-\varnothing}^{*}, W_{,,-s}^{*}:=W_{-\varnothing,-\{s\}}^{*}$, and $W_{-b,-s}^{*}:=W_{-\{b\},-\{s\}}^{*}$. Buyer $b \in \mathbb{B}$ and seller $s \in \mathbb{S}$ are complements if

$$
W_{-b, .}^{*}-W_{-b,-s}^{*}+W_{.,-s}^{*}-W_{-b,-s}^{*} \leq W^{*}-W_{-b,-s}^{*}
$$

The expressions $W_{-b, .}^{*}-W_{-b,-s}^{*}$ and $W_{.,-s}^{*}-W_{-b,-s}^{*}$ capture, respectively, the individual marginal contribution to the social gains from trade of seller $s$ and buyer $b$ when they are added to an allocation problem that consists of all buyers other than $b$ and all sellers other than $s$. The right side is the marginal contribution of adding the pair consisting of $b$ and $s$ to the allocation problem without this pair. Thus, the inequality simply states that the marginal contribution of the pair is not less than the sum of the individual marginal contributions when the other agent is not there. An equivalent representation of the complements condition can be found by adding $W^{*}$ to both

\footnotetext{
${ }^{16}$ Imposing monotonicity in a model with free disposal is without loss of generality because a buyer can dispose of any object in his package that reduces his utility and a seller can produce a larger package and dispose of some objects if this is less costly than producing a smaller package.

${ }^{17}$ If $X^{*}(\mathbf{v}, \mathbf{c})$ contains multiple elements, $x^{*}(\mathbf{v}, \mathbf{c})$ can be chosen arbitrarily from among them.
} 
sides of this inequality and rearranging:

$$
W^{*}-W_{-b, .}^{*}+W^{*}-W_{.,-s}^{*} \geq W^{*}-W_{-b,-s}^{*}
$$

We use this alternative representation in the rest of the paper. It states that the sum of the individual marginal products exceeds the marginal product of the pair.

Following Holmström (1979), we say the type space is smoothly connected if for all buyers $b$, the set of mappings $\left\{u_{b}\left(\cdot, \mathbf{v}_{b}\right): X \rightarrow \mathbb{R} \mid \mathbf{v}_{b} \in V_{b}\right\}$ is smoothly connected, and for all sellers $s$, the set of mappings $\left\{k_{s}\left(\cdot, \mathbf{c}_{s}\right): X \rightarrow \mathbb{R} \mid \mathbf{c}_{s} \in C_{s}\right\}$ is smoothly connected. ${ }^{18}$ We assume that the type space $V \times C$ is smoothly connected.

We also assume that for every $b \in \mathbb{B}$, there exists a least efficient type $\underline{\mathbf{v}}_{b} \in V_{b}$ that does not trade in any efficient allocation, and similarly for sellers, where we let $\overline{\mathbf{c}}_{s} \in C_{s}$ denote the least efficient type for seller $s \in \mathbb{S}$.

A direct mechanism is a triple $\left(\chi, \mathbf{t}^{\beta}, \mathbf{t}^{\sigma}\right)$, where $\chi: V \times C \rightarrow X$ is the allocation rule and $\mathbf{t}^{\beta}: V \times C \rightarrow \mathbb{R}^{B}$ and $\mathbf{t}^{\sigma}: V \times C \rightarrow \mathbb{R}^{S}$ are the payment rules. Thus, given reports $(\mathbf{v}, \mathbf{c}), \chi(\mathbf{v}, \mathbf{c})$ is the allocation, buyer $b$ pays $t_{b}^{\beta}(\mathbf{v}, \mathbf{c})$, and seller $s$ receives $t_{s}^{\sigma}(\mathbf{v}, \mathbf{c})$. An allocation rule is efficient if it selects an efficient allocation for every $(\mathbf{v}, \mathbf{c})$. For the purpose of deriving the conditions under which efficiency is impossible without running a deficit, the well-known revelation principle (Myerson, 1981) implies that we can restrict attention to direct mechanisms without loss of generality.

A mechanism $\left(\chi, \mathbf{t}^{\beta}, \mathbf{t}^{\sigma}\right)$ is dominant strategy incentive compatible (DIC) if for each buyer $b$, type profile $(\mathbf{v}, \mathbf{c})$, and type $\hat{\mathbf{v}}_{b}$ for buyer $b$,

$$
u_{b}\left(\chi_{b}(\mathbf{v}, \mathbf{c}), \mathbf{v}_{b}\right)-t_{b}^{\beta}(\mathbf{v}, \mathbf{c}) \geq u_{b}\left(\chi_{b}\left(\hat{\mathbf{v}}_{b}, \mathbf{v}_{-b}, \mathbf{c}\right), \mathbf{v}_{b}\right)-t_{b}^{\beta}\left(\hat{\mathbf{v}}_{b}, \mathbf{v}_{-b}, \mathbf{c}\right)
$$

and for each seller $s$, type profile $(\mathbf{v}, \mathbf{c})$, and type $\hat{\mathbf{c}}_{s}$ for seller $s$,

$$
t_{s}^{\sigma}(\mathbf{v}, \mathbf{c})-k_{s}\left(\chi_{s}(\mathbf{v}, \mathbf{c}), \mathbf{c}_{s}\right) \geq t_{s}^{\sigma}\left(\mathbf{v}, \hat{\mathbf{c}}_{s}, \mathbf{c}_{-s}\right)-k_{s}\left(\chi_{s}\left(\mathbf{v}, \hat{\mathbf{c}}_{s}, \mathbf{c}_{-s}\right), \mathbf{c}_{s}\right)
$$

where $\chi_{b}$ and $\chi_{s}$ denote the packages that buyer $b$ receives and seller $s$ produces under allocation rule $\chi$. Given a type profile $(\mathbf{v}, \mathbf{c})$, the revenue $R(\mathbf{v}, \mathbf{c})$ generated by a mechanism with transfers $\left(\mathbf{t}^{\beta}, \mathbf{t}^{\sigma}\right)$ is

$$
R(\mathbf{v}, \mathbf{c})=\sum_{b \in \mathbb{B}} t_{b}^{\beta}(\mathbf{v}, \mathbf{c})-\sum_{s \in \mathbb{S}} t_{s}^{\sigma}(\mathbf{v}, \mathbf{c})
$$

\footnotetext{
${ }^{18}$ For a formal definition of smooth connectedness, see Definition 1 in Holmström (1979).
} 
A mechanism satisfies ex post individual rationality (EIR) if for each buyer $b$ and type profile $(\mathbf{v}, \mathbf{c}), u_{b}\left(\chi_{b}(\mathbf{v}, \mathbf{c}), \mathbf{v}_{b}\right)-t_{b}^{\beta}(\mathbf{v}, \mathbf{c}) \geq 0$, and for each seller $s$ and type profile $(\mathbf{v}, \mathbf{c}), t_{s}^{\sigma}(\mathbf{v}, \mathbf{c})-$ $k_{s}\left(\chi_{s}(\mathbf{v}, \mathbf{c}), \mathbf{c}_{s}\right) \geq 0$.

We say that a mechanism is efficient if its allocation rule $\chi(\mathbf{v}, \mathbf{c})$ is efficient. We say that efficient trade is impossible if all efficient mechanisms satisfying DIC and EIR have $R(\mathbf{v}, \mathbf{c}) \leq 0$ for all $(\mathbf{v}, \mathbf{c})$, with a strict inequality for some $(\mathbf{v}, \mathbf{c})$.

\section{Background: Impossibility of Efficient Trade}

In this section, we synthesize results from the literature and use them to derive an impossibility result for one-to-one trading environments.

To start, it is well known that if the condition

$$
\sum_{b \in \mathbb{B}}\left[W^{*}-W_{-b, .}^{*}\right]+\sum_{s \in \mathbb{S}}\left[W^{*}-W_{\cdot,-s}^{*}\right] \geq W^{*}
$$

is satisfied for all $(\mathbf{v}, \mathbf{c})$, with a strict inequality for some $(\mathbf{v}, \mathbf{c})$, then efficient trade is impossible. The condition appears, in slightly different forms, in McAfee (1991), Makowski and Mezzetti (1994), Williams (1999), Yenmez (2015), and Krishna (2002). Our statement of condition (2) explicitly reflects the two-sided nature of the allocation problems we study.

Lemma 1 Efficient trade is impossible if (2) holds for all $(\mathbf{v}, \mathbf{c})$ and with a strict inequality for some $(\mathbf{v}, \mathbf{c})$.

For completeness, we provide a formal proof in the Appendix. ${ }^{19}$ Here, we develop the intuition that will be used to extend the impossibility theorem to assignment-representable problems in Section 4 .

Observe first that $W^{*}-W_{-b, .}^{*}$ is the marginal product of buyer $b$ and $W^{*}-W_{.,-s}^{*}$ is the marginal product of seller $s$. Interpreted in this way, condition (2) says that the sum of marginal products exceeds the total product $W^{*}$. Because incentive compatibility requires that every agent be paid his marginal product ${ }^{20}$ condition (2) implies a deficit. The proof shows that this intuition is correct. It additionally uses the uniqueness of Groves' schemes when the type space is smoothly connected

\footnotetext{
${ }^{19}$ As becomes clear from the proof of Lemma 1 , efficient trade is possible if $\sum_{b \in \mathbb{B}}\left[W^{*}-W_{-b, .}^{*}\right]+\sum_{s \in \mathbb{S}}\left[W^{*}-W_{.,-s}^{*}\right] \leq$ $W^{*}$ holds for all $(\mathbf{v}, \mathbf{c})$; see Yenmez $(2015)$ for an analysis along these lines.

${ }^{20}$ Makowski and Ostroy (1987) show that any VCG mechanism is equivalent to a "MP mechanism" that always gives each agent his marginal product, plus perhaps a lump sum.
} 
(Holmström, 1979) and the fact that the VCG mechanism is the revenue maximizing mechanism among efficient mechanisms that satisfy DIC and EIR. ${ }^{21,22}$

As noted, our focus is on dominant strategy incentive compatibility, while much of the literature considers Bayesian incentive compatibility. Our approach rests on the insight that, for setups like ours in which every agent has a least efficient type who never trades, if (2) is satisfied for all $(\mathbf{v}, \mathbf{c})$, with a strict inequality for some $(\mathbf{v}, \mathbf{c})$, then efficient trade is impossible in any efficient mechanism that is Bayesian incentive compatible, provided distributions are independent and have full support. This insight is based on Theorem 3 of Williams (1999). In this sense, our approach comes with little loss of generality and allows us to abstract from distributional assumptions. ${ }^{23}$

Segal and Whinston (2016) define the marginal core as the set of total payoff vectors such that no coalition involving all but one agent can profitably deviate. They show that the nonemptiness of the marginal core, along with some regularity conditions, implies that any efficient mechanism runs a deficit. The nonemptiness of the marginal core is equivalent to condition (2). ${ }^{24}$ Yenmez (2015) uses the converse inequality of Segal and Whinston's condition to prove the existence of an

\footnotetext{
${ }^{21}$ While the VCG mechanism is named after the independent contributions by Vickrey (1961), Clarke (1971), and Groves (1973), the genealogy of the term is interesting and seems, to us, still somewhat unclear. To the best of our knowledge, Makowski and Ostroy (1987) were the first to use it. Green and Laffont (1977) seem to have coined the term Groves schemes in honor of the seminal contribution by Groves (1973), which specializes to VCG with an appropriately chosen constant. Mas-Colell et al. (1995, Section 23.C) refer to what we call VCG as a Groves-Clarke scheme. Tracing out the origins of the mechanism is not only complicated by the fact that it has not been introduced by anyone deliberately (as opposed to, e.g., Nash equilibrium), but also by the circumstance that the environment for which Groves schemes were first introduced (Groves, 1973) was too rich to permit dominant strategies. Only when simplified to the setup studied in Groves and Loeb (1975) does the mechanism endow agents with dominant strategies. More importantly, there appear to be conflicting definitions of VCG that coincide when there exist least efficient types but differ when such types do not exist.

${ }^{22}$ There is an interesting parallel between how (2) implies impossibility of efficient trade and how increasing returns to scale in the theory of the firm implies that a firm cannot break even under price-taking behavior. In either case, agents are paid their marginal products, whose sum exceeds the total product.

${ }^{23}$ Admittedly, our approach is restrictive in that we abstract from interdependent values. Our focus on private values is not only in line with much of the literature on the possibility or impossibility of efficient trade, but also based on the insight, due to Jehiel and Moldovanu (2001), that with multi-dimensional types, efficient, incentive-compatible allocation may be impossible with or without running a deficit when two-stage mechanisms as in Mezzetti (2004) are not admissible. In a sense, this makes the two-sided allocation problem with private values the pertinent one. Moreover, even when efficient two-stage mechanisms can be used, whether or not they run a budget deficit depends on the fine details of the model, such as the distributions. In contrast, our impossibility theorem only requires least efficient types for every agent who never trade under efficiency, independence, and positive densities on the support; otherwise, we do not impose any restriction on the distributions. That said, our approach is limited in that we do not allow for shared ownership. As shown by Cramton et al. (1987) for a single object that is jointly owned by ex ante symmetric agents, there is an efficient, incentive compatible, and interim individually rational mechanism if ownership is sufficiently symmetric. As noted by Fieseler et al. (2003), this result does not necessarily extend to interdependent values because of the simultaneous presence of the winner's and the loser's curse. With or without interdependent values, extending our already rich model to also allow for shared ownership is unlikely to yield clearcut results because of the intertwined connection between the distributions of types, ownership shares, and payoff functions.

${ }^{24}$ The marginal core is multi-valued if (2) is satisfied with a strict inequality, single-valued if it is satisfied with an equality, and empty if it is not satisfied.
} 
efficient DIC-EIR mechanism that does not run a deficit.

Less well understood are the conditions on the primitives of the allocation problem under which condition (2) holds. For example, (2) is satisfied in the bilateral trade problem of Myerson and Satterthwaite (1983). Assuming identical supports, the marginal product is $W^{*}$ for both the buyer and the seller whenever trade occurs, implying that the sum of the marginal products is $2 W^{*}$. In the remainder of the paper, we derive conditions under which (2) is satisfied.

We begin by showing that condition (2) is satisfied in all allocation problems in which each buyer trades with at most one seller and each seller trades with at most one buyer. Such problems with one-to-one trading are natural when agents' types are one-dimensional, buyers have unit demand, sellers have unit capacities, and objects are homogeneous; however, they are not confined to setups with one-dimensional types. For example, the housing market model of Shapley and Shubik (1972) has multi-dimensional types because buyers with unit demand have heterogeneous values for the sellers' houses.

In what the subsequent literature has referred to as an assignment game, Shapley (1962) derives a result - stated as Lemma 2 below - that implies that buyers and sellers are complements in an assignment game. We use this result to show that in all allocation problems involving one-to-one trading, efficient trade is impossible.

In order to formally state Shapley's result, we require the following definitions: An assignment game assumes one-to-one matching between two sides of the "market" (say, buyers and sellers). It consists of a $B \times S$-dimensional value matrix

$$
A=\left(\begin{array}{cccc}
a_{1,1} & a_{1,2} & \cdots & a_{1, S} \\
a_{2,1} & a_{2,2} & \cdots & a_{2, S} \\
\vdots & \vdots & \ddots & \vdots \\
a_{B, 1} & a_{B, 2} & \cdots & a_{B, S}
\end{array}\right),
$$

whose entries $a_{b, s}$ give the value if buyer $b$ and seller $s$ are matched. A matching is then defined by a $B \times S$-dimensional matching matrix $L$, whose elements $l_{b, s} \in\{0,1\}$ indicate whether buyer $b$ and seller $s$ are matched together, with $l_{b, s}=1(0)$ meaning they are (not). A matching is feasible if the sum of any row or column in the matching matrix is at most one. A feasible matching is efficient if it maximizes the value

$$
\sum_{b \in \mathbb{B}} \sum_{s \in \mathbb{S}} l_{b, s} a_{b, s}
$$

That is, the optimal matching from an assignment game can be found by solving the following 
linear programming problem: ${ }^{25}$

$$
\begin{aligned}
\max _{L} \sum_{b \in \mathbb{B}} \sum_{s \in \mathbb{S}} l_{b, s} a_{b, s} \\
\text { subject to } \quad \sum_{s \in \mathbb{S}} l_{b, s} \leq 1 \quad \text { for all } \quad b \in \mathbb{B} \\
\text { and } \quad \sum_{b \in \mathbb{B}} l_{b, s} \leq 1 \quad \text { for all } \quad s \in \mathbb{S} .
\end{aligned}
$$

We denote the value generated from an optimal matching by $V^{*}(A)$. Similarly, we let $V^{*}\left(A_{-\mathbb{I},-\mathbb{J}}\right)$ be the maximal value of an assignment game when rows $\mathbb{I} \subseteq \mathbb{B}$ and columns $\mathbb{J} \subseteq \mathbb{S}$ are removed.

In analogy to (1), a buyer $b$ and a seller $s$ are complements to one another in an assignment game if their joint value added, $V^{*}(A)-V^{*}\left(A_{-b,-s}\right)$, is smaller than the sum of their individual values added, which are $V^{*}(A)-V^{*}\left(A_{-b, .}\right)$ for the buyer and $V^{*}(A)-V^{*}\left(A_{.,-s}\right)$ for the seller. That is, $b$ and $s$ are complements if

$$
V^{*}(A)-V^{*}\left(A_{-b, .}\right)+V^{*}(A)-V^{*}\left(A_{.,-s}\right) \geq V^{*}(A)-V^{*}\left(A_{-b,-s}\right) .
$$

Similarly, two buyers $b$ and $b^{\prime}$ (or two sellers $s$ and $s^{\prime}$ ) are substitutes for each other if their joint value added exceeds the sum of their individual values added:

$$
\begin{aligned}
& V^{*}(A)-V^{*}\left(A_{-b, .}\right)+V^{*}(A)-V^{*}\left(A_{-b^{\prime}, .}\right) \leq V^{*}(A)-V^{*}\left(A_{-\left\{b, b^{\prime}\right\}, .}\right) \\
& V^{*}(A)-V^{*}\left(A_{.,-s}\right)+V^{*}(A)-V^{*}\left(A_{.,-s^{\prime}}\right) \leq V^{*}(A)-V^{*}\left(A_{.,-\left\{s, s^{\prime}\right\}}\right) .
\end{aligned}
$$

Shapley (1962) establishes the following result:

Lemma 2 (Shapley, 1962) In any assignment game, any two agents on the same side of the market are substitutes for each other, while any two agents on opposite sides are complements to each other.

For any allocation problem in which the efficient allocation is one-to-one for all $(\mathbf{v}, \mathbf{c})$, a buyer $b$ and a seller $s$ who are matched always trade the package that maximizes their joint surplus, whose value we denote by $a_{b, s}:=\max \left\{\max _{x \in \mathbb{P}_{s}} u_{b}\left(x, \boldsymbol{v}_{b}\right)-k_{s}\left(x, \boldsymbol{c}_{s}\right), 0\right\}$. Using these elements to construct an assignment game value matrix $A$ as in (3), it follows that the allocation problem can be represented as an assignment game.

At an optimal matching in the assignment game, we have $W^{*}-W_{-b, .}^{*}=V^{*}(A)-V^{*}\left(A_{-b, .}\right)$, $W^{*}-W_{.,-s}^{*}=V^{*}(A)-V^{*}\left(A_{.,-s}\right)$, and $W^{*}-W_{-b,-s}^{*}=V^{*}(A)-V^{*}\left(A_{-b,-s}\right)$. By Lemma 2, buyers

\footnotetext{
${ }^{25}$ Solutions to this problem can be found by inspection if $A$ is small enough, by using the Hungarian algorithm of Kuhn (1955), or through linear programming techniques (Dantzig, 1963).
} 
and sellers in the assignment game are complements. In conjunction with the above relationship between $W^{*}$ and $V^{*}$, this result implies that for any $b \in \mathbb{B}$ and $s \in \mathbb{S}$, the complements condition (1) is satisfied. In other words, buyers and sellers in the underlying allocation problem are complements.

Let $T \geq 0$ be the number of trading buyer-seller pairs under efficiency and, if $T>0$, relabel agents so that buyer $b_{\tau}$ trades with seller $s_{\tau}$ for $\tau=1, \ldots, T$. If a buyer and a seller who optimally trade together are both removed, the optimal matching does not change for the remaining agents. Summing up the right side of (1) over $\tau$ thus yields $\sum_{\tau=1}^{T}\left(W^{*}-W_{-b_{\tau},-s_{\tau}}^{*}\right)=W^{*}$. Because the marginal product of every agent who does not trade is zero (e.g., if $b$ does not trade, $W^{*}-W_{-b, .}^{*}=0$ ), we can likewise sum up the left side of (1) to obtain

$$
\sum_{\tau=1}^{T}\left[W^{*}-W_{-b_{\tau}, .}^{*}+W^{*}-W_{.,-s_{\tau}}^{*}\right]=\sum_{b \in \mathbb{B}}\left[W^{*}-W_{-b, .}^{*}\right]+\sum_{s \in \mathbb{S}}\left[W^{*}-W_{.,-s}^{*}\right],
$$

which is the left side of (2). Consequently, in any allocation problem with one-to-one trading, (1) implies (2). In addition, for any buyer $b$ and seller $s$, (1), and thus (2), holds strictly for the type profile $\mathbf{v}_{-b}=\underline{\mathbf{v}}_{-b}$ and $\mathbf{c}_{-s}=\overline{\mathbf{c}}_{-s}$ and $\mathbf{v}_{b}$ and $\mathbf{c}_{s}$ such that $b$ and $s$ efficiently trade with each other and no other trades occur-because $b$ and $s$ are the only traders, the sum of their marginal products strictly exceeds the maximum social gains from trade. We summarize this in the following proposition.

Proposition 1 Efficient trade is impossible in any allocation problem with one-to-one trading.

By formalizing arguments outlined in Loertscher et al. (2015), Proposition 1 generalizes the impossibility result stated there beyond the homogeneous goods model. It also goes beyond existing impossibility results by providing conditions on payoff functions (or the trading technology) such that impossibility obtains. It implies that if buyers and sellers have unit demand and unit supply (such as in the model of Shapley and Shubik (1972) and much of the mechanism design literature) or are exogenously restricted to a single trading partner, efficient trade is impossible. Thus, the intuition that the complementarity between the buyer and the seller in a bilateral trade problem leads to the impossibility of efficient trade extends to any allocation problem involving one-to-one trading.

Because (1) is a pairwise property, summing up over trading pairs in one-to-one trading problem yields (2) and thereby Proposition 1 . When trade is not restricted to be one-to-one, this line of 
logic does not directly extend because, even when all buyer-seller pairs are complements, groups of buyers and sellers can be substitutes. In what follows, we provide conditions on primitives that guarantee that overall, agents are complements.

\section{Assignment-Representable Problems}

Having synthesized existing results on impossibility and shown their connection to Shapley (1962), we now build upon these insights to identify a general class of two-sided allocation problems in which the complementarity between buyers and sellers is maintained. We begin in Section 4.1 by defining an assignment-representable allocation problem. In Section 4.2, we establish the relation between an assignment-representable allocation problem and the assignment game that represents it. In Section 4.3, we generalize Shapley (1962) to show that (2) holds in any assignment-representable allocation problem, which establishes the impossibility result for any such problem. Conditions on primitives for a two-sided allocation problem to be an assignment-representable problem are then the focus of Section 5.

\subsection{Definitions}

Consider an assignment game with a value matrix $A$ of dimension $(B+1) O \times O$. The rows of the value matrix represent unit constituents of the buyers and sellers, while the columns of the matrix represent objects. To allow for the matrix to encode the assignment of any feasible package to a buyer, each buyer in the original allocation problem is represented in the matrix as $O$ unit constituents. There are $O$ additional rows to represent the unit constituents of the sellers, resulting in a total of $(B+1) O$ rows. Each column of the matrix represents one of the $O$ objects.

Let $\hat{\mathbb{B}}$ be the set of buyer unit constituents with cardinality $\hat{B}=B \cdot O$. Let $\hat{\mathbb{S}}$ be the set of seller unit constituents with cardinality $\hat{S}=O$. Then $\hat{\mathbb{B}} \cup \hat{\mathbb{S}}$ is the set of all unit constituents, with typical element $r$ (" $r$ " for row). The cardinality of this set, $(B+1) O$, is equal to the number of rows of the value matrix.

It is useful to also define $\hat{\mathbb{B}}_{b} \subseteq \hat{\mathbb{B}}$ to be the set of unit constituents of buyer $b$ with cardinality $O$. Likewise we define $\hat{\mathbb{S}}_{s} \subseteq \hat{\mathbb{S}}$ to be the set of unit constituents of seller $s$ with cardinality $O_{s}$. We also define the set of unit constituents $\hat{\mathbb{I}}$ to be the unit constituents corresponding to the buyers in

set $\mathbb{I} \subseteq \mathbb{B}$, i.e., $\hat{\mathbb{I}}:=\cup_{b \in \mathbb{I}} \hat{\mathbb{B}}_{b}$. The set of seller unit constituents $\hat{\mathbb{J}}:=\cup_{s \in J} \hat{\mathbb{S}}_{s}$ corresponds to sellers in set $\mathbb{J} \subseteq \mathbb{S}$ in an analogous way. Finally, we denote by $\mathbb{O}_{\mathbb{J}}:=\cup_{s \in \mathbb{J}} \mathbb{O}_{s}$ the set of objects in the 
potential sets of the sellers in set $\mathbb{J}$.

Definition 1 A unit constituent-object assignment game of a two-sided allocation problem consists of $a(B+1) O \times O$ matrix $A=\left(a_{r, o}\right)_{r \in \hat{\mathbb{B}} \cup \hat{\mathbb{S}}, o \in \mathbb{O}}$ with nonnegative elements satisfying $a_{r, o}=0$ for all $(r, o)$ such that $r \in \hat{\mathbb{S}}_{s}$ and $o \in \mathbb{O}_{s^{\prime}}$ with $s \neq s^{\prime}$ and $a(B+1) O \times O$ matching matrix $L$ with elements $l_{r, o} \in\{0,1\}$.

As an illustration of how a two-sided allocation problem may be represented as a unit constituentobject assignment game, consider the following example. Suppose there are two buyers, David and Martin, and one seller, Lloyd. Lloyd can produce two heterogeneous objects, $A$ and $B$, at cost 2 for $A, 3$ for $B$, and 6 for producing both $A$ and $B$. David values $A$ at $9, B$ at 5 , and the package $A B$ at 12, while Martin's values are 5, 6, and 10, respectively. Efficiency cannot be achieved using oneto-one trades because the efficient allocation assigns Lloyd's two objects to two different buyers, with object $A$ going to David and object $B$ to Martin. However, one can create unit constituents of the agents in such a way that a corresponding assignment game exists, as shown in Figure 1(a).

$\underline{\text { Panel (a) }}$

\begin{tabular}{l|c|c|}
\multicolumn{1}{c}{} & \multicolumn{1}{c}{$A$} & \multicolumn{1}{c}{$B$} \\
\cline { 2 - 3 } David-1st unit & $\mathbf{9}$ & 5 \\
\cline { 2 - 3 } David-2nd unit & 7 & 3 \\
\cline { 2 - 3 } Martin-1st unit & 5 & $\mathbf{6}$ \\
\cline { 2 - 3 } Martin-2nd unit & 4 & 5 \\
\cline { 2 - 3 } Lloyd-1st unit & 3 & 4 \\
\cline { 2 - 3 } Lloyd-2nd unit & 2 & 3 \\
\cline { 2 - 3 } & &
\end{tabular}

Panel (b)

\begin{tabular}{l|c|c|}
\multicolumn{1}{c}{} & \multicolumn{1}{c}{$A$} & \multicolumn{1}{c}{$B$} \\
\cline { 2 - 3 } Martin-1st unit & 5 & $\mathbf{6}$ \\
\cline { 2 - 3 } Martin-2nd unit & $\mathbf{4}$ & 5 \\
\cline { 2 - 3 } Lloyd-1st unit & 3 & 4 \\
\cline { 2 - 3 } Lloyd-2nd unit & 2 & 3 \\
\cline { 2 - 3 } & &
\end{tabular}

Panel (c)

\begin{tabular}{l|c|c|}
\multicolumn{1}{c}{} & \multicolumn{1}{c}{$A$} & \multicolumn{1}{c}{$B$} \\
\cline { 2 - 3 } David-1st unit & $\mathbf{9}$ & 5 \\
\cline { 2 - 3 } David-2nd unit & 7 & 3 \\
\cline { 2 - 3 } Lloyd-1st unit & 3 & $\mathbf{4}$ \\
\cline { 2 - 3 } Lloyd-2nd unit & 2 & 3 \\
\cline { 2 - 3 } & &
\end{tabular}

Figure 1: Unit constituent-object assignment game.

For the buyers, the first unit entries are the stand-alone utilities from $A$ and $B$, while the second unit entries are the marginal utilities from adding $A$ or $B$ to the package that already includes the other object. For the seller, the second unit entries are the marginal costs of producing either $A$ or $B$, while the first unit entries are the incremental costs of producing either $A$ or $B$ given that the other one is produced.

The assignment of an object to a buyer's unit constituent means that the buyer receives the object, while the assignment of an object to a seller's unit constituent means that the seller does not produce the object. Thus, if no unit constituent of a seller receives an object, the interpretation is that the seller produces both objects. 
The solution to this assignment game, shown in bold in Figure 1(a), assigns object $A$ to the first unit constituent of David and $B$ to the first unit constituent of Martin, while no unit constituent of Lloyd receives an object, meaning that Lloyd produces both objects. The total value from this assignment is 15. It differs from the maximum social gains from trade of 9 in the two-sided allocation problem by 6 , which is the cost imposed on the seller to produce both objects.

In the two-sided allocation problem underlying a unit constituent-object assignment game, objects could be produced by a seller and allocated to a buyer or could not be produced. These allocations are expressed in the matching matrix in the following way. When an object $o_{s} \in \mathbb{O}_{s}$ is assigned to a unit constituent of buyer $b$, this implies that the object is produced by seller $s$ and traded to buyer $b$. When an object $o_{s} \in \mathbb{O}_{s}$ is assigned to a unit constituent of seller $s$, this implies that the object is not produced. In this way, the assignment game encodes an analogue of the twosided allocation problem, where objects are assigned to buyer unit constituents for consumption or to seller unit constituents to reduce required production. For buyer unit constituents, the value matrix encodes the utility that the buyer receives from consuming an object. For seller unit constituents, the matrix encodes the cost savings from not having to produce an object. The restriction in Definition 1 that $a_{r, o}=0$ if $r \in \hat{\mathbb{S}}_{s}$ and $o \in \mathbb{O}_{s^{\prime}}$ with $s \neq s^{\prime}$ embeds the constraint that sellers can only produce objects from their own potential set.

The maximum value created by the unit constituent-object assignment game with value matrix $A$ can be calculated by solving the following linear programming problem:

$$
\begin{aligned}
V^{*}(A):=\max _{L} \sum_{r \in \hat{\mathbb{B}} \cup \hat{\mathbb{S}}} \sum_{o \in \mathbb{O}} l_{r, o} a_{r, o} & \\
\text { subject to } & \sum_{o \in \mathbb{O}} l_{r, o} \leq 1 \quad \text { for all } \quad r \in \hat{\mathbb{B}} \cup \hat{\mathbb{S}} \\
\text { and } & \sum_{r \in \hat{\mathbb{B}} \cup \hat{\mathbb{S}}} l_{r, o} \leq 1 \quad \text { for all } \quad o \in \mathbb{O} .
\end{aligned}
$$

It will be useful to define the assignment game in which a subset of unit constituents and a subset of objects have been removed. For any $\hat{\mathbb{T}} \subseteq \hat{\mathbb{B}} \cup \hat{\mathbb{S}}$, and $\mathbb{K} \subseteq \mathbb{O}$, let $A_{-\hat{\mathbb{T}},-\mathbb{K}}$ be defined from matrix $A$ by removing all rows relating to unit constituents in $\hat{\mathbb{T}}$ and all columns relating to objects in $\mathbb{K}^{26}$

\footnotetext{
${ }^{26}$ Notice that $A_{-\hat{\mathbb{N}},-\mathbb{K}}$ will not necessarily satisfy Definition 1 . In fact $A_{-\hat{\mathbb{N}},-\mathbb{K}}$ is a unit constituent-object assignment game of a two-sided allocation problem if and only if for some $\mathbb{I} \subseteq \mathbb{B}$ and $\mathbb{J} \subseteq \mathbb{S}, \hat{\mathbb{T}}=\hat{\mathbb{I}} \cup \hat{\mathbb{J}}$ and $\mathbb{K}=\mathbb{O}_{\mathbb{J}}$. That is, for each buyer either none or all of his unit constituents are removed and for each seller either none or all of his unit constituents and objects are removed. Observe however that the maximum value of any assignment game, whether or not it satisfies Definition 1, can be computed using (4).
} 
For any unit constituent-object assignment game with value matrix $A$ and any allocation $x \in X$, a feasible matching $L$ is said to be isomorphic to $x$ if for any $o \in \mathbb{O}$,

$$
\begin{aligned}
& \sum_{r \in \hat{\mathbb{B}}_{b}} l_{r, o}=\left\{\begin{array}{ll}
1, & \text { if } o \in x_{b} \\
0, & \text { otherwise }
\end{array} \quad \text { for all } b \in \mathbb{B} \quad\right. \text { and } \\
& \sum_{r \in \hat{\mathbb{S}}_{s}} l_{r, o}=\left\{\begin{array}{ll}
1, & \text { if } o \in \mathbb{O}_{s} \backslash x_{s} \\
0, & \text { otherwise }
\end{array} \quad \text { for all } s \in \mathbb{S} .\right.
\end{aligned}
$$

When $L$ is isomorphic to $x$, unit constituents of each buyer $b$ are jointly assigned $x_{b}$ and unit constituents of each seller $s$ are jointly assigned $\mathbb{O}_{s} \backslash x_{s}$, which are the objects that $s$ does not have to produce under allocation $x$. Let $\mathbb{L}(A, x)$ be the set of feasible matchings of $A$ that are isomorphic to $x$ and $\mathbb{L}^{*}(A, x) \subseteq \mathbb{L}(A, x)$ be the set of matchings that are isomorphic to $x$ and return the largest value. We refer to $\mathbb{L}^{*}(A, x)$ as the set of best isomorphic matchings of allocation $x$ in value matrix $A$. By focusing on the largest value matchings, we maintain equivalence with the original optimization problem because, given an agent and the objects in his package, the unit constituents are assigned to the objects in the best possible way. ${ }^{27}$ Formally, we define $\mathbb{L}^{*}(A, x)$ to be the set of all $L \in \mathbb{L}(A, x)$ such that for all $L^{\prime} \in \mathbb{L}(A, x)$,

$$
\sum_{r \in \hat{\mathbb{B}} \cup \hat{\mathbb{S}}} \sum_{o \in \mathbb{O}} l_{r, o} a_{r, o} \geq \sum_{r \in \hat{\mathbb{B}} \cup \hat{\mathbb{S}}} \sum_{o \in \mathbb{O}} l_{r, o}^{\prime} a_{r, o} .
$$

Where there is no risk of confusion, we write $L^{x}$ for a best isomorphic matching of $x$ in $A$. If $\mathbb{L}^{*}(A, x)$ contains more than one element, then $L^{x}$ can be arbitrarily chosen among them. It is also useful to define $x^{0}$ to be the allocation where nothing is produced $\left(x_{b}^{0}=\varnothing\right.$ for all $b$ and $x_{s}^{0}=\varnothing$ for all $s)$. Let $L^{x^{0}} \in L^{*}\left(A, x^{0}\right)$ be a best isomorphic matching of $x^{0}$. Notice that $L^{x^{0}}$ matches all unit constituents of each seller to an object in that seller's potential set but leaves all buyer unit constituents unmatched.

We can now formally define an assignment-representable problem:

Definition 2 A two-sided allocation problem is assignment representable if for all ( $\mathbf{v}, \mathbf{c})$ there exists a unit constituent-object assignment game satisfying Definition 1 such that for any feasible

\footnotetext{
${ }^{27}$ Given an agent and the objects in his package, there may be multiple ways to assign the objects to the agent's unit constituents. To guarantee that the two maximization problems lead to the same result, we require that the unit constituents be assigned the objects in a way that is optimal for the agent. If one did not focus on the largest value matchings, Lemma 3 below would no longer hold.
} 
allocation $x \in X$ and any best isomorphic matching $L^{x}$,

$$
\begin{aligned}
& u_{b}\left(x_{b}, \mathbf{v}_{b}\right)=\sum_{r \in \hat{\mathbb{B}}_{b}} \sum_{o \in \mathbb{O}} l_{r, o}^{x} a_{r, o} \quad \text { for all } b \in \mathbb{B} \quad \text { and } \\
& k_{s}\left(x_{s}, \mathbf{c}_{s}\right)=\sum_{r \in \hat{\mathbb{S}}_{s}} \sum_{o \in \mathbb{O}}\left[l_{r, o}^{x^{0}} a_{r, o}-l_{r, o}^{x} a_{r, o}\right] \quad \text { for all } s \in \mathbb{S} .
\end{aligned}
$$

A two-sided allocation problem is assignment representable if all the information needed to describe an allocation, the social gains from trade of an allocation, and the resulting trade network can be encoded in the optimal solution of an assignment game between unit constituents and objects. The utility that a buyer receives from consuming a package equals the sum of the values generated from assigning that package to unit constituents of that buyer. Likewise, for any seller, there is an exact correspondence between the cost of producing a package and the value that is generated from objects not assigned to unit constituents of that seller.

\subsection{Relation between Allocation Problems and Assignment Games}

We now show that the correspondence between an assignment-representable allocation problem and the assignment game that represents it is general insofar as it also holds when subsets of buyers and sellers (and their corresponding unit constituents and objects) are removed. To see this, consider a two-sided allocation problem that is assignment representable. Remove a subset of buyers $\mathbb{I} \subseteq \mathbb{B}$, a subset of sellers $\mathbb{J} \subseteq \mathbb{S}$, and the objects that correspond to these sellers $\mathbb{O}_{\mathbb{J}}$. Let $x=\left(\left(x_{b}\right)_{b \in \mathbb{B} \backslash \mathbb{I}},\left(x_{s}\right)_{s \in \mathbb{S} \backslash \mathbb{I}}\right)$ be an allocation in this smaller two-sided allocation problem. Let $A_{-\hat{\mathbb{I}} \cup \hat{\mathbb{J}},-\mathbb{O}_{\mathbb{J}}}$ be a submatrix of $A$ with the rows corresponding to unit constituents not in $\hat{\mathbb{I}} \cup \hat{\mathbb{J}}$ and the columns corresponding to objects not in $\mathbb{O}_{\mathfrak{J}}$. Note that $A_{-\hat{\mathbb{I}} \cup \hat{\mathbb{J}},-\mathbb{O}_{\mathbb{J}}}$ continues to satisfy Definitions 1 and 2 for any allocation $x$. This implies that the smaller two-sided allocation problem is assignment representable and has $A_{-\hat{\mathbb{I}} \cup \hat{\mathbb{J}},-\mathbb{O}_{\mathbb{J}}}$ as the value matrix of the corresponding assignment game. ${ }^{28} \mathrm{We}$ use this correspondence in the following lemma, which implies that the social gains from trade in a best isomorphic matching are equal to the maximal social gains from trade generated from the corresponding assignment game, up to a constant.

Lemma 3 Consider an assignment-representable problem with types $(\mathbf{v}, \mathbf{c})$. For any subsets of agents $\mathbb{I} \subseteq \mathbb{B}$ and $\mathbb{I} \subseteq \mathbb{S}$,

$$
W_{-\mathbb{I},-\mathbb{I}}^{*}=V^{*}\left(A_{-\hat{\mathbb{I}} \cup \hat{\mathbb{J}},-\mathbb{O}_{\mathbb{J}}}\right)-V^{*}\left(A_{-\hat{\mathbb{B}} \cup \hat{\mathbb{I}},-\mathbb{O}_{\mathbb{J}}}\right) .
$$

\footnotetext{
${ }^{28}$ There may be multiple alternative assignment games that correspond to the two-sided allocation problem. We concentrate on the one obtained by removing rows and columns from our original matrix $A$.
} 
The term $V^{*}\left(A_{-\hat{\mathbb{B}} \cup \hat{\mathbb{J}},-\mathbb{O}_{\mathbb{J}}}\right)$ in $(5)$ captures the value of the assignment game with only sellers $\mathbb{S} \backslash \mathbb{J}$ and objects $\mathbb{O} \backslash \mathbb{O}_{\mathbb{J}}$ present (and no buyers), in which case the optimal matching assigns to every unit constituent of seller $s$ an element of $\mathbb{O}_{s}$. The term normalizes the right side in (5) to zero when, given the value matrix $A_{-\hat{\mathbb{I}} \cup \hat{\mathbb{J}},-\mathbb{O}_{\mathbb{J}}}$, it is optimal to assign all the objects to unit constituents of sellers. In the underlying allocation problem, this corresponds to a situation in which it is efficient not to trade.

Lemma 3 allows us to relate the marginal product of buyers and sellers to the values generated from a best isomorphic matching in the assignment game. To see this, first note that the normalization term for $V^{*}(A)$ and $V^{*}\left(A_{-\hat{\mathbb{B}}_{b}, .}\right)$ are the same for all $b$ and thus Lemma 3 implies that

$$
\sum_{b \in \mathbb{B}}\left[W^{*}-W_{-b, .}^{*}\right]=\sum_{b \in \mathbb{B}}\left[V^{*}(A)-V^{*}\left(A_{-\hat{\mathbb{B}}_{b}, .}\right)\right]
$$

where $A_{-\hat{\mathbb{B}}_{b},}:=A_{-\hat{\mathbb{B}}_{b},-\varnothing}$. Second, by carefully accounting for the normalization terms, we show in the proof of Lemma 4 in the Appendix that

$$
\sum_{s \in \mathbb{S}}\left[W^{*}-W_{.,-s}^{*}\right]-W^{*}=\sum_{s \in \mathbb{S}}\left[V^{*}(A)-V^{*}\left(A_{-\hat{\mathbb{S}}_{s},-\mathbb{O}_{s}}\right)\right]-V^{*}(A) .
$$

These two equations imply that the difference between the sum of marginal products and the maximum social gains from trade in the two-sided allocation problem,

$$
\sum_{b \in \mathbb{B}}\left[W^{*}-W_{-b, .}^{*}\right]+\sum_{s \in \mathbb{S}}\left[W^{*}-W_{.,-s}^{*}\right]-W^{*}
$$

is equal to

$$
\sum_{b \in \mathbb{B}}\left[V^{*}(A)-V^{*}\left(A_{-\hat{\mathbb{B}}_{b}, .}\right)\right]+\sum_{s \in \mathbb{S}}\left[V^{*}(A)-V^{*}\left(A_{-\hat{\mathbb{S}}_{s},-\mathbb{O}_{s}}\right)\right]-V^{*}(A) .
$$

The equality between (6) and (7) is the basis for the following lemma:

Lemma 4 An assignment-representable problem satisfies (2) if and only if

$$
\sum_{b \in \mathbb{B}}\left[V^{*}(A)-V^{*}\left(A_{-\hat{\mathbb{B}}_{b}, .}\right)\right]+\sum_{s \in \mathbb{S}}\left[V^{*}(A)-V^{*}\left(A_{-\hat{\mathbb{S}}_{s},-\mathbb{O}_{s}}\right)\right] \geq V^{*}(A)
$$

and satisfies (2) strictly if and only if (8) holds strictly.

Before moving on to our main impossibility theorem, it is useful to revisit our example involving David, Martin, and Lloyd to highlight the correspondence between assignment games and their underlying allocation problem and to demonstrate how Lemma 3 naturally leads to Lemma 4. 
In our example, the buyer and seller sets can be written as: $\mathbb{B}=\{D, M\}$ and $\mathbb{S}=\{\mathcal{L}\}$. The set of objects is $\mathbb{O}=\{A, B\}$. Using Definition 2, the two-sided allocation problem in our example is assignment representable if the associated unit constituent-object assignment game shown in Figure 1(a) is such that for any feasible allocation $x$ and any best isomorphic matching $L^{x}$, the following three equalities hold: first for the buyers,

$$
\begin{aligned}
& u_{D}\left(x_{D}, \mathbf{v}_{D}\right)=l_{D_{1}, A}^{x} 9+l_{D_{2}, A}^{x} 7+l_{D_{1}, B}^{x} 5+l_{D_{2}, B}^{x} 3 \text { and } \\
& u_{M}\left(x_{M}, \mathbf{v}_{M}\right)=l_{M_{1}, A}^{x} 5+l_{M_{2}, A}^{x} 4+l_{M_{1}, B}^{x} 6+l_{M_{2}, B}^{x} 5
\end{aligned}
$$

and, second, for the seller,

$$
k_{\mathcal{L}}\left(x_{\mathcal{L}}, \mathbf{c}_{\mathcal{L}}\right)=\underbrace{\left(l_{\mathcal{L}_{1}, A}^{x^{0}} 3+l_{\mathcal{L}_{1}, B}^{x^{0}} 4+l_{\mathcal{L}_{2}, A}^{x^{0}} 2+l_{\mathcal{L}_{2}, B}^{x^{0}}\right)}_{=6}-\left(l_{\mathcal{L}_{1}, A}^{x} 3+l_{\mathcal{L}_{1}, B}^{x} 4+l_{\mathcal{L}_{2}, A}^{x} 2+l_{\mathcal{L}_{2}, B}^{x} 3\right),
$$

where the two unit constituents for each agent are denoted with subscripts 1 and 2 and where $L^{x^{0}}$, the matching matrix associated with no production, can be defined either as having $l_{\mathcal{L}_{1}, A}^{x^{0}}=$ $l_{\mathcal{L}_{2}, B}^{x^{0}}=1$ or $l_{\mathcal{L}_{1}, B}^{x^{0}}=l_{\mathcal{L}_{2}, A}^{x^{0}}=1$.

To verify that the assignment game is represented by the matrix in Figure 1(a), we can check that the three equalities above hold for each potential allocation. For example, if we consider the allocation $x=(\{A, B\}, \varnothing,\{A, B\})$, then the left sides of the three equations are equal to 12,0 , and 6 , respectively. There are two best isomorphic matchings, one with $l_{D_{1}, A}^{x}=l_{D_{2}, B}^{x}=1$ and one with $l_{D_{1}, B}^{x}=l_{D_{2}, A}^{x}=1$. In either case, the right sides of the three equations are equal to 12,0 , and 6 , respectively, and thus the equalities are satisfied.

To illustrate the need to use a best isomorphic matching, consider the allocation $x=(\{A\}, \varnothing,\{A\})$. Then the left sides of the three equations are equal to 9,0 , and 2 . There are four feasible matchings that are isomorphic to $x$, namely the matchings given by $l_{D_{1}, A}^{x}=l_{\mathcal{L}_{1}, B}^{x}=1, l_{D_{1}, A}^{x}=l_{\mathcal{L}_{2}, B}^{x}=1$, $l_{D_{2}, A}^{x}=l_{\mathcal{L}_{1}, B}^{x}=1$, and $l_{D_{2}, A}^{x}=l_{\mathcal{L}_{2}, B}^{x}=1$, but only the first of these, which corresponds to David's first unit constituent being assigned object $A$ and Lloyd's first unit constituent being assigned object $B$, is a best isomorphic matching. Using this best isomorphic matching, the right sides of the three equations are equal to 9,0 , and $6-4=2$, the same as for the allocation problem.

Repeating this exercise for each allocation $x$ and corresponding best isomorphic matching, one can show that our example is assignment representable.

We now verify Lemma 3 and show how it leads to Lemma 4. In the allocation problem of our example,

$$
W^{*}=9, W_{-D, .}^{*}=4, W_{-M, .}^{*}=7, \text { and } W_{.,-\mathcal{L}}^{*}=0 .
$$


Lemma 3, applied to our example, states that

$$
\begin{aligned}
W^{*} & =V^{*}(A)-V^{*}\left(A_{-\left\{D_{1}, D_{2}, M_{1}, M_{2}\right\}, .}\right) \\
W_{-D, .}^{*} & =V^{*}\left(A_{-\left\{D_{1}, D_{2}\right\}, .}\right)-V^{*}\left(A_{-\left\{D_{1}, D_{2}, M_{1}, M_{2}\right\}, .}\right) \\
W_{-M, .}^{*} & =V^{*}\left(A_{-\left\{M_{1}, M_{2}\right\}, .}\right)-V^{*}\left(A_{-\left\{D_{1}, D_{2}, M_{1}, M_{2}\right\}, .}\right) \\
W_{.,-\mathcal{L}}^{*} & =V^{*}\left(A_{-\left\{\mathcal{L}_{1}, \mathcal{L}_{2}\right\},-\{A, B\}}\right)-V^{*}\left(A_{-\left\{D_{1}, D_{2}, M_{1}, M_{2}, \mathcal{L}_{1}, \mathcal{L}_{2}\right\},-\{A, B\}}\right),
\end{aligned}
$$

as well as additional equalities (with both sides equal to zero) associated with both buyers being excluded or individual buyers being excluded along with the seller. To see that these equalities hold, note that as illustrated in Figure $1(\mathrm{a}), V^{*}(A)=15$, as illustrated in Figure $1(\mathrm{~b}), V^{*}\left(A_{-\left\{D_{1}, D_{2}\right\}, .}\right)=$ 10, and as illustrated in Figure $1(\mathrm{c}), V^{*}\left(A_{-\left\{M_{1}, M_{2}\right\}, .}\right)=13$. With both buyers excluded, we have $V^{*}\left(A_{-\left\{D_{1}, D_{2}, M_{1}, M_{2}\right\}, .}\right)=6$. Thus, the right sides of the first three equalities above are equal to $15-6=9,10-6=4$, and $13-6=7$, matching the left sides. Eliminating the seller and his associated objects, $V^{*}\left(A_{-\left\{\cdot, \mathcal{L}_{1}, \mathcal{L}_{2}\right\},-\{A, B\}}\right)=0$, so the fourth equality above also holds, being equal to zero on both sides.

We now illustrate Lemma 4. In the allocation problem of our example, the sum of the agents' marginal products minus the maximum social gains from trade is

$$
\underbrace{\left(W^{*}-W_{-D, .}^{*}\right)}_{=9-4}+\underbrace{\left(W^{*}-W_{-M, .}^{*}\right)}_{=9-7}+\underbrace{\left(W^{*}-W_{.,-\mathcal{L}}^{*}\right)}_{=9-0}-\underbrace{W^{*}}_{=9}=7 .
$$

Thus, because this is positive, condition (2) is satisfied. Using Lemma 3, the analogous expression for the unit constituent-object assignment game is

$$
\underbrace{\left(V^{*}(A)-V^{*}\left(A_{-\left\{D_{1}, D_{2}\right\}, .}\right)\right)}_{=15-10}+\underbrace{\left(V^{*}(A)-V^{*}\left(A_{-\left\{M_{1}, M_{2}\right\}, .}\right)\right)}_{=15-13}+\underbrace{\left(V^{*}(A)-V^{*}\left(A_{-\left\{\mathcal{L}_{1}, \mathcal{L}_{2}\right\},-\{A, B\}}\right)\right)}_{=15-0}-\underbrace{V^{*}(A)}_{=15},
$$

which equals 7 and is thus the same as for the allocation problem.

\subsection{Impossibility of Efficient Trade in Assignment-Representable Problems}

We now show the following:

Theorem 1 Efficient trade is impossible in any assignment-representable problem.

Given Lemma 4, in order to prove Theorem 1, it is sufficient to show that (8) is satisfied in any unit constituent-object assignment game (and strictly for some type vector). Shapley's (1962) 
result (our Lemma 2) and our next result, which generalizes Shapley's, prove useful for this step by ensuring that unit constituents are substitutes for each other, which implies that the sum of their marginal products exceeds the marginal product of the agent.

For an assignment game between unit constituents and objects, we say that unit constituents are set substitutes if for any two disjoint subsets of unit constituents $\hat{\mathbb{T}} \subseteq \hat{\mathbb{B}} \cup \hat{\mathbb{S}}$ and $\hat{\mathbb{T}}^{\prime} \subseteq \hat{\mathbb{B}} \cup \hat{\mathbb{S}}$ with $\hat{\mathbb{T}} \cap \hat{\mathbb{T}}^{\prime}=\varnothing$,

$$
V^{*}(A)-V^{*}\left(A_{-\hat{\mathbb{T}}_{,} .}\right)+V^{*}(A)-V^{*}\left(A_{-\hat{\mathbb{T}}^{\prime}, .}\right) \leq V^{*}(A)-V^{*}\left(A_{-\hat{\mathbb{T}} \cup \hat{\mathbb{T}}^{\prime}, .}\right) .
$$

We say that any collection of unit constituents are substitutes if for any subset of unit constituents $\hat{\mathbb{T}} \subseteq \hat{\mathbb{B}} \cup \hat{\mathbb{S}}$

$$
\sum_{r \in \hat{\mathbb{T}}}\left[V^{*}(A)-V^{*}\left(A_{-r, .}\right)\right] \leq V^{*}(A)-V^{*}\left(A_{-\hat{\mathbb{T}}, .}\right) .
$$

Likewise, objects are set substitutes if for any two disjoint subsets of objects $\mathbb{K} \subseteq \mathbb{O}$ and $\mathbb{K}^{\prime} \subseteq \mathbb{O}$ with $\mathbb{K} \cap \mathbb{K}^{\prime}=\varnothing$,

$$
V^{*}(A)-V^{*}\left(A_{.,-}, \mathbb{K}\right)+V^{*}(A)-V^{*}\left(A_{.,-}-\mathbb{K}^{\prime}\right) \leq V^{*}(A)-V^{*}\left(A_{.,-} \mathbb{K}_{\mathbb{K}^{\prime}}\right),
$$

and any collection of objects are substitutes if for any subset of objects $\mathbb{K} \subseteq \mathbb{O}$,

$$
\sum_{o \in \mathbb{K}}\left[V^{*}(A)-V^{*}\left(A_{.,-o}\right)\right] \leq V^{*}(A)-V^{*}\left(A_{.,-\mathbb{K}}\right) .
$$

In addition, unit constituents and objects are set complements if for any $\hat{\mathbb{T}} \subseteq \hat{\mathbb{B}} \cup \hat{\mathbb{S}}$ and $\mathbb{K} \subseteq \mathbb{O}$,

$$
V^{*}(A)-V^{*}\left(A_{-\hat{\mathbb{T}}, .}\right)+V^{*}(A)-V^{*}\left(A_{.,-\mathbb{K}}\right) \geq V^{*}(A)-V^{*}\left(A_{-\hat{\mathbb{T}},-\mathbb{K}}\right)
$$

Lemma 5 For any assignment game between unit constituents and objects, (i) unit constituents are set substitutes, (ii) any collection of unit constituents are substitutes, (iii) objects are set substitutes, (iv) any collection of objects are substitutes, and (v) unit constituents and objects are set complements.

As shown in the proof of Theorem 1 in the Appendix, Shapley's (1962) result, together with its generalization in Lemma 5 (specifically parts (ii)-(iv)) and a careful accounting of matches between objects and sellers, allows one to show that (8) is satisfied in any unit constituent-object assignment game. Thus, using Lemma 4, efficient trade is impossible in all assignment-representable problems. Interestingly, while complementarity between sellers and buyers remains at the heart of the argument, the proof also relies on our generalization of Shapley's substitutes condition. 


\section{Characterization of Assignment-Representable Problems}

In this section, we first characterize assignment-representable problems by showing that an allocation problem is assignment representable if and only if each agent is decomposable, that is, his payoff function can be derived as the solution to a unit constituent-object assignment game. Then, we introduce a new family of payoff functions exhibiting what we call size-dependent discounts (SDD) and show that SDD implies decomposability. Finally, we discuss the relations between SDD, decomposability, and substitutes payoffs.

\subsection{Decomposition}

For a buyer $b \in \mathbb{B}$ with type $\mathbf{v}_{b}$, consider an assignment game between unit constituents and objects with an $O \times O$ value matrix $A^{b}$. Each row of $A^{b}$ represents a unit constituent of $b$ and each column represents an object. Let $V^{*}\left(A^{b}\right)$ be the maximal value that can be generated from the assignment game. Then $A^{b}$ is a decomposition of buyer $b$ with type $\mathbf{v}_{b}$ if for any package $x_{b} \in \mathbb{P}$,

$$
u_{b}\left(x_{b}, \mathbf{v}_{b}\right)=V^{*}\left(A_{x_{b}}^{b}\right)
$$

where $A_{x_{b}}^{b}:=A_{.,-\mathbb{O} \backslash x_{b}}^{b}$, that is, $A_{x_{b}}^{b}$ is the submatrix of $A^{b}$ containing exclusively its columns related to objects in $x_{b}$. In other words, a decomposition of a buyer exists if it is possible to construct an assignment game whose maximal value coincides with the buyer's utility function for any package of objects. Buyer $b$ is decomposable if such a decomposition exists for every type $\mathbf{v}_{b}$. This definition is equivalent to that of assignment valuations (Ostrovsky and Paes Leme, 2015), that is, a buyer is decomposable if and only if his utility function is an assignment valuation. ${ }^{29}$ The term decomposability highlights that when agents can be decomposed into unit constituents, then the allocation problem can be turned into an assignment game between unit constituents and objects. ${ }^{30}$ It also points to a connection between assignment games and the ideas of Hurwicz

\footnotetext{
${ }^{29}$ Assignment valuations (AV) are a special case of the endowment assignment valuations (EAV) introduced by Hatfield and Milgrom (2005) and used by Ostrovsky and Paes Leme (2015). If all agents' payoff functions are EAV, then the allocation problem can also be represented as a series of one-to-one matches and our impossibility theorem also holds. The main difference between EAV and AV is that EAV introduces "null" objects that can only be assigned to constituents of a particular agent. Because in our setting such objects do not relate naturally to the actual allocation, we have chosen to work with the stricter, but more intuitive, condition.

${ }^{30}$ Aizerman and Malishevski (1981) introduce a decomposition concept that is different in nature to ours. They show that a choice function can be decomposed into a union of individual choice functions that each picks one object if and only if it is path-independent (see their Theorem 3). These individual choice functions can be thought of as unit constituents. In contrast to our concept of unit constituents, these individual choice functions operate independently from one another, and the same object can be picked by multiple ones. Chambers and Yenmez (2017) build upon Theorem 3 of Aizerman and Malishevski (1981) to show that many-to-many matching problems without transfers can be turned into one-to-one problems whenever agents have path-independent choice functions.
} 
(1973), who uses the term decomposable to refer to environments without externalities. Indeed, because an agent is decomposable if his payoff function is the solution to a unit constituent-object assignment game, decomposability in our setting means that there are no externalities between unit constituents of the same agent. ${ }^{31}$ Importantly, as we discuss below, decomposability does not exclude the possibility that the payoff function itself exhibits externalities.

We extend the concept of decomposability to sellers. The $O_{s} \times O_{s}$ value matrix $A^{s}$ is a decomposition of seller $s \in \mathbb{S}$ with type $\mathbf{c}_{s}$ if for any package $x_{s} \in \mathbb{P}_{s}$ :

$$
k_{s}\left(x_{s}, \mathbf{c}_{s}\right)=V^{*}\left(A^{s}\right)-V^{*}\left(A_{\mathbb{O}_{s} \backslash x_{s}}^{s}\right)
$$

where $A_{\mathbb{O}_{s} \backslash x_{s}}^{s}:=A_{.,-x_{s}}^{s}$, the submatrix of $A^{s}$ containing exclusively the columns related to objects in $\mathbb{O}_{s} \backslash x_{s}$. Seller $s$ is decomposable if such a decomposition exists for each type $\mathbf{c}_{s}{ }^{32}$

Our decomposition of sellers turns a two-sided allocation problem between buyers and sellers into a one-sided allocation problem between agents and objects. Each object can be assigned to either a buyer (or one of his unit constituents) in order to increase his utility, or to a seller (or one of his unit constituents) in order to decrease his cost. This treats agents on both sides symmetrically and vastly simplifies the problem. If all agents are decomposable, then their decompositions can be stacked in order to obtain a unit constituent-object assignment game that satisfies Definition 1, which implies that the two-sided allocation problem is assignment representable. In an assignmentrepresentable allocation problem, the efficient allocation corresponds to the optimal matching in the unit constituent-object assignment game. If some agent is not decomposable, then a unit constituent-object assignment game cannot be constructed.

Proposition 2 A two-sided allocation problem is assignment representable if and only if each buyer and seller is decomposable.

Proposition 2 and Theorem 1 imply the impossibility of efficient trade when all buyers and sellers are decomposable, or equivalently have assignment valuations. To our knowledge, this is the first instance in which the, in our view surprising, connection between the impossibility of efficient trade and assignment valuations is recognized.

\footnotetext{
${ }^{31}$ There are no externalities in the unit constituent-object assignment game because any unit constituent's valuation for a given object is independent of the valuations of any other unit constituent. Of course, there is still rivalry in consumption between unit constituents because every object can only be allocated once.

${ }^{32}$ We have restricted the number of unit constituents to be equal to the maximum number of objects that an agent can consume or produce. This is without loss of generality, as shown in Lemma 6 at the end of the Appendix.
} 


\subsection{Size-dependent Discounts Payoff Functions}

Proposition 2 explicitly states a condition on the primitives of the model for the impossibility of efficient trade; importantly this condition can be verified individually for each agent. However, it leaves open the question of what are the families of payoff functions that ensure decomposability. Although a complete characterization of decomposability is beyond the scope of this paper, we now provide a simple sufficient condition for decomposability that is satisfied in a wide array of economically relevant models.

To develop a sense of what is sufficient, it is useful to begin with payoff functions that are additive in the stand-alone values of individual objects. Specifically, buyer $b$ has an additive payoff function if for any package $x$ and arbitrary ordering of the objects $\left\{o_{1}, \ldots, o_{O}\right\}$ in $x, u_{b}\left(x, \mathbf{v}_{b}\right)=$ $\sum_{m=1}^{O} u_{b}\left(\left\{o_{m}\right\}, \mathbf{v}_{b}\right)$. As mentioned in the introduction, agents with additive payoff functions are obviously decomposable. Notice also that with additive payoffs, externalities are not only absent at the level of unit constituents, but also at the level of the agent: a buyer's willingness to pay for an object is independent of whatever other goods he may or may not be allocated.

One can depart substantively from additive payoffs and no externalities at the level of the agent's payoff function without losing decomposability. As a first point of departure, consider the homogeneous goods model with nonincreasing marginal values. The homogeneous goods assumption along with free disposal means that the stand-alone utility of every object is a nonnegative constant, that is, $u_{b}\left(\left\{o_{i}\right\}, \mathbf{v}_{b}\right)=v \geq 0$ for all $o_{i} \in \mathbb{O}$, while nonincreasing marginal values means that $u_{b}\left(x, \mathbf{v}_{b}\right)=\sum_{j=1}^{|x|} \max \left\{v-\delta_{j}, 0\right\}$, where the $\delta_{j}$ 's are "discounts" that satisfy $0=\delta_{1} \leq \delta_{2} \leq \ldots$, reflecting the decrease in marginal values as the size of the package $x$ increases. Clearly, there are externalities at the agent's payoff level whenever $\delta_{j}>0$ for some $j$ because then the willingness to pay for the $j$-th object depends on whether the agent is allocated other objects as well. Nevertheless, homogeneous goods and nonincreasing marginal values ensure decomposability.

These two specifications, which at first sight differ starkly, can be relaxed (and recombined) further. A first and natural extension of the homogeneous goods model is an additively separable version of what Ausubel (2006) terms a heterogeneous commodity model. Let $\left\{\mathbb{O}_{m}\right\}_{m=1}^{M}$ be a partition of $\mathbb{O}$ comprised of $M$ blocks (or "commodities"), and suppose that within each block a buyer views objects as homogeneous and has nonincreasing marginal values for them. ${ }^{33}$ That is, if

\footnotetext{
${ }^{33}$ Following set theory terminology, we call an element of a partition a block. Blocks may reflect commodities, as in Ausubel's model, or may be specific to each agent.
} 
buyer $b$ consumes a package $x_{m}$ that consists exclusively of objects in the $m$-th block, his utility is

$$
u_{b}\left(x_{m}, \mathbf{v}_{b}\right)=\sum_{i=1}^{\left|x_{m}\right|} \max \left\{v_{m}-\delta_{m, i}, 0\right\}
$$

where $v_{m}$ is the stand-alone utility of objects in the $m$-th block (i.e., $u_{b}\left(\left\{o_{m}\right\}, \mathbf{v}_{b}\right)=v_{m}$ for all $\left.o_{m} \in \mathbb{O}_{m}\right)$ and the $\delta_{m, i}$ 's are block-specific size-dependent discounts that satisfy $0=\delta_{m, 1} \leq \delta_{m, 2} \leq$ ..., reflecting the nonincreasing nature of marginal values. Additive separability then means that if buyer $b$ consumes $x=\cup_{m} x_{m}$, his utility is

$$
u_{b}\left(x, \mathbf{v}_{b}\right)=\sum_{m=1}^{M} u_{b}\left(x_{m}, \mathbf{v}_{b}\right)=\sum_{m=1}^{M} \sum_{i=1}^{\left|x_{m}\right|} \max \left\{v_{m}-\delta_{m, i}, 0\right\} .
$$

Given that the homogeneous goods payoff functions ensure decomposability, not surprisingly so do heterogeneous commodities payoff functions of the additively separable form.

The homogeneous goods model can independently be extended to incorporate heterogeneity in stand-alone values when the assumption of block-specific size-dependent discounts is maintained. To fix ideas, assume for now that there is only one block and let $x \subseteq \mathbb{O}$ be $b$ 's consumption bundle. Then let $o_{(i)}(x)$ be buyer $b$ 's $i$-th most preferred object in this bundle according to the objects' stand-alone values for a given $\mathbf{v}_{b}$, that is,

$$
u_{b}\left(\left\{o_{(1)}(x)\right\}, \mathbf{v}_{b}\right) \geq u_{b}\left(\left\{o_{(2)}(x)\right\}, \mathbf{v}_{b}\right) \geq \ldots \geq u_{b}\left(\left\{o_{(|x|)}(x)\right\}, \mathbf{v}_{b}\right)
$$

where to conserve notation we do not indicate the dependence of $o_{(i)}(x)$ on $b$ and $\mathbf{v}_{b}$. If buyer $b$ has such heterogeneous stand-alone values and size-dependent discounts satisfying $0=\delta_{1} \leq \delta_{2} \leq \ldots$, then his utility from consuming $x$ is

$$
u_{b}\left(x, \mathbf{v}_{b}\right)=\sum_{i=1}^{|x|} \max \left\{u_{b}\left(\left\{o_{(i)}(x)\right\}, \mathbf{v}_{b}\right)-\delta_{i}, 0\right\} .
$$

For similar reasons as in the homogeneous goods model, this type of payoff function, which combines the richness of the stand-alone values admitted by additive payoffs with a notion of decreasing marginal values typically associated with the homogeneous goods model, also ensures that the agent is decomposable. Of course, nothing in this construction requires there to be only one block. Our definition of size-dependent discounts below allows for an arbitrary number of blocks; as a result it identifies a family of payoff functions that nests additive payoffs, homogeneous goods, and the additively separable version of Ausubel's model. 
Definition 3 Buyer b's utility function exhibits SDD if for each type $\mathbf{v}_{b}$ there exists a partition $\left\{\mathbb{O}_{m}\right\}_{m=1}^{M}$ and a collection of discount vectors $\boldsymbol{\delta}=\left\{\boldsymbol{\delta}_{1}, \ldots, \boldsymbol{\delta}_{M}\right\}$ with $0=\delta_{m, 1} \leq \delta_{m, 2} \leq \cdots \leq$ $\delta_{m, O_{m}}$ for all $m \in\{1, \ldots, M\}$ such that for any $x \in \mathbb{P}$,

$$
u_{b}\left(x, \mathbf{v}_{b}\right)=\sum_{m=1}^{M} \sum_{i=1}^{\left|x_{m}\right|} \max \left\{u_{b}\left(\left\{o_{(i)}\left(x_{m}\right)\right\}, \mathbf{v}_{b}\right)-\delta_{m, i}, 0\right\}
$$

where for all $m \in\{1, \ldots, M\}, x_{m}:=x \cap \mathbb{O}_{m}$.

SDD requires (i) that a buyer's utility function be additive across blocks in a partition of the set of objects and (ii) that within each block, the value of a package be equal to the sum of the stand-alone utilities minus a total discount that depends on the number of units in the block that the package contains.

Analogous to the buyers, given a subset of objects $x \subseteq \mathbb{O}_{s}$, denote the object in this subset with the $i$-th lowest stand-alone cost for seller $s$, as $o_{(i)}(x)$.

Definition 4 Seller s's cost function exhibits SDD if for each type $\mathbf{c}_{s}$ there exists a partition $\left\{\mathbb{O}_{m, s}\right\}_{m=1}^{M}$ and collection of discount vectors $\boldsymbol{\delta}=\left\{\boldsymbol{\delta}_{1}, \ldots, \boldsymbol{\delta}_{M}\right\}$ with $0=\delta_{m, 1} \leq \delta_{m, 2} \leq \cdots \leq$ $\delta_{m, O_{m, s}}$ for all $m \in\{1, \ldots, M\}$ such that for any $x \in \mathbb{P}_{s}$,

$$
k_{s}\left(x, \mathbf{c}_{s}\right)=\sum_{m=1}^{M} \sum_{i=1}^{\left|x_{m}\right|}\left(k_{s}\left(\left\{o_{(i)}\left(x_{m}\right)\right\}, \mathbf{c}_{s}\right)+\delta_{m, i}\right),
$$

where $x_{m}:=x \cap \mathbb{O}_{m, s}$.

A seller's cost function exhibits SDD if there exists a partition of the seller's potential set where (i) costs are additive across blocks, and (ii) within each block, the cost of producing a package is equal to the sum of the stand-alone costs plus a total increment that only depends on the number of units of the block that the package contains. Such preferences may represent a situation in which a seller has $M$ production lines or factories and each production facility produces homogeneous goods at nondecreasing marginal costs.

By construction, the family of SDD payoff functions nests a wide range of payoff functions as special cases. As mentioned earlier, if all stand-alone utilities (costs) are the same, then the buyer (seller) considers the objects to be homogeneous. If the discount (increment) parameters are very large then buyers (sellers) are unit traders in the sense that they efficiently consume, 
respectively produce, packages containing at most one unit. ${ }^{34}$ The assignment game of Shapley and Shubik (1972) is a special case of this model because buyers are unit traders and the potential set of each seller contains one object. In contrast, if the discount (increment) parameters are zero or if each block contains exactly one object, then the agent has additive payoffs. The version of Ausubel's (2006) model in which payoffs are additively separable across commodities satisfies SDD. Although additive separability is more restrictive than the model Ausubel studies, as mentioned, SDD permits heterogeneity in stand-alone values of objects within each commodity (or block), which Ausubel's model does not.

The following results show that the general nature of SDD payoff functions allows us to classify various well-known models as assignment-representable problems.

Theorem 2 An agent is decomposable if his payoff function exhibits SDD.

Corollary 1 Any two-sided allocation problem in which all agents' payoff functions exhibit SDD is assignment representable.

An immediate implication of Corollary 1 is that efficient trade is impossible in any two-sided allocation problem in which all payoff functions exhibit SDD. Because decomposability is determined at the individual level, Corollary 1 allows us to derive the impossibility of efficient trade for setups in which agents have different payoff functions, as long as all of them exhibit SDD.

\subsection{Size-Dependent Discounts, Decomposability, and Substitutes Payoffs}

We conclude this section by analyzing the relation between size-dependent discounts, decomposability, and substitutes payoffs. We have defined size-dependent discounts and decomposability above, so we begin by defining substitutes payoffs.

For a buyer $b$ with type $\mathbf{v}_{b}$ consuming a package $x \in \mathbb{P}$, two disjoint subsets of that package $y, z \subseteq x$ with $y \cap z=\varnothing$ are substitutes for each other if

$$
u_{b}\left(x, \mathbf{v}_{b}\right)-u_{b}\left(x \backslash y, \mathbf{v}_{b}\right)+u_{b}\left(x, \mathbf{v}_{b}\right)-u_{b}\left(x \backslash z, \mathbf{v}_{b}\right) \leq u_{b}\left(x, \mathbf{v}_{b}\right)-u_{b}\left(x \backslash(y \cup z), \mathbf{v}_{b}\right),
$$

that is, if the joint marginal utility of $y$ and $z$ is greater than or equal to the sum of their individual marginal utilities. This definition specializes to Shapley's definition of substitutes if $y$ and $z$ each

\footnotetext{
${ }^{34}$ Allocation problems involving one-to-one trading can be mapped into an assignment-representable problem with SDD payoff functions by first determining the package that maximizes the joint surplus of each pair and then redefining cost and utility functions over these efficient packages. Agents in this transformed problem are unit traders.
} 
only contain one object. For a seller $s$ with type $\mathbf{c}_{s}$ producing a package $x \in \mathbb{P}_{s}$, two disjoint subsets of that package $y, z \subseteq x$ with $y \cap z=\varnothing$ are substitutes for each other if the joint marginal cost of $y$ and $z$ is less than or equal to the sum of their individual marginal costs:

$$
k_{s}\left(x, \mathbf{c}_{s}\right)-k_{s}\left(x \backslash y, \mathbf{c}_{s}\right)+k_{s}\left(x, \mathbf{c}_{s}\right)-k_{s}\left(x \backslash z, \mathbf{c}_{s}\right) \geq k_{s}\left(x, \mathbf{c}_{s}\right)-k_{s}\left(x \backslash(y \cup z), \mathbf{c}_{s}\right) .
$$

We say that an agent has substitutes payoffs if for any possible type and package, any two disjoint subsets of that package are substitutes for one another.

In all the applications introduced above, buyers and sellers had substitutes payoffs. This is not a coincidence. The following proposition shows that decomposability is a stricter condition than substitutes payoffs and that SDD is a stricter condition than decomposability.

Proposition 3 (i) SDD implies decomposability. (ii) Decomposability implies substitutes payoffs. (iii) The converse statements are false if and only if there are three or more objects.

Part (i) of Proposition 3 is a restatement of Theorem 2 and therefore does not require a separate proof. Part (ii) reflects a result first reported by Hatfield and Milgrom (2005, Theorem 14) for endowment assignment valuations. ${ }^{35}$ For completeness, and because our definition of substitutes payoffs differs from theirs and applies to sellers as well, we provide a proof of part (ii) in the Appendix.

We now turn to part (iii) and first show that the converse statements hold if there are only two objects, say $o_{1}$ and $o_{2} \cdot{ }^{36}$ Consider a buyer. By the definition of substitutes payoffs:

$$
u_{b}\left(\left\{o_{1}, o_{2}\right\}, \mathbf{v}_{b}\right)-u_{b}\left(\left\{o_{1}\right\}, \mathbf{v}_{b}\right)+u_{b}\left(\left\{o_{1}, o_{2}\right\}, \mathbf{v}_{b}\right)-u_{b}\left(\left\{o_{2}\right\}, \mathbf{v}_{b}\right) \leq u_{b}\left(\left\{o_{1}, o_{2}\right\}, \mathbf{v}_{b}\right),
$$

where the normalization $u_{b}\left(\varnothing, \mathbf{v}_{b}\right)=0$ has been used. By setting $\delta=u_{b}\left(\left\{o_{1}\right\}, \mathbf{v}_{b}\right)+u_{b}\left(\left\{o_{2}\right\}, \mathbf{v}_{b}\right)-$ $u_{b}\left(\left\{o_{1}, o_{2}\right\}, \mathbf{v}_{b}\right)$, which is nonnegative because objects are substitutes, we obtain the size-dependent discount. An analogous reasoning holds for a seller. Thus, with only two objects, substitutes payoffs imply SDD, which by part (ii) implies decomposability.

We are left to show that with more than two objects, the converse statements of (i) and (ii) are not true. We do this by adding a third object to our earlier example.

Recall from that example that David had stand-alone utilities of 9 and 5 for objects $A$ and $B$ and derived a utility of 12 from the package $A B$. Suppose now that, in addition, there is a third

\footnotetext{
${ }^{35}$ Ostrovsky and Paes Leme (2015, Theorem 1) show that substitutes, as defined by Hatfield and Milgrom (2005), do not imply endowment assignment valuations. Chen et al. (2014) discuss additional restrictions on the EAV model that ensures strong substitutes, as defined by Echenique and Oviedo (2006).

${ }^{36}$ The converse statements hold trivially with only one object.
} 
object whose stand-alone utility for David is 7, while his utilities from packages $A C, B C$, and $A B C$ are 14,11 , and 16 , respectively. It is easy to verify that David has substitutes payoffs.

We now show that David is not decomposable. In order to represent David's utility function as a unit constituent-object assignment game, we need to find values $a, b, c, d, e$, and $f$ in Figure 2(a) such that David's utility corresponds to the solution of the assignment game for any subset of objects. When looking only at the package $A B$, we require that either (i) $a=7$ and $b \leq 3$ or (ii) $a \leq 7$ and $b=3$. In both of these cases, we must set $c=6$ in order for the package $B C$ to have a value of 11 . However, this is incompatible with a value of only 14 for $A C$. Thus, David is not decomposable. ${ }^{37}$ This result extends trivially to any larger number of objects because decomposability requires that the equality between the utility and the value of the assignment game hold for all subsets of objects.

\begin{tabular}{|c|c|c|c|c|c|c|c|}
\hline \multicolumn{2}{|c|}{ Panel (a) } & & & \multicolumn{4}{|c|}{ Panel (b) } \\
\hline \multirow{4}{*}{$\begin{array}{l}\text { David - 1st unit } \\
\text { David - 2nd unit } \\
\text { David - 3rd unit }\end{array}$} & $A$ & $B$ & $C$ & \multirow{4}{*}{$\begin{array}{l}\text { David - 1st unit } \\
\text { David - 2nd unit } \\
\text { David - 3rd unit }\end{array}$} & $A$ & $B$ & C \\
\hline & 9 & 5 & 7 & & 9 & 5 & 7 \\
\hline & $a$ & $b$ & $c$ & & 8 & 3 & 5 \\
\hline & $d$ & $e$ & $f$ & & 7 & 2 & 4 \\
\hline
\end{tabular}

Figure 2: Decompositions of David with three objects.

Figure 2(b) provides an example in which David is decomposable but his payoff function does not exhibit SDD. In this example, David's utilities are 9, 5, and 7 for $A, B$, and $C$, respectively. Further, his utilities for packages $A B, A C, B C$, and $A B C$ are 13,15, 10, and 17. SDD requires the existence of a single discount that applies to all packages of two objects. However, the discount for assembling a package consisting of objects $A B$ is 1 , while the discount for assembling the package consisting of objects $B C$ is 2 . Thus, in this case, we have decomposability but not SDD. ${ }^{38}$ This example can be extended to any larger number of objects as follows. For packages of two objects, set the discount to 1 if the package contains $A$ and to 2 otherwise. Then set $\delta_{3} \leq \delta_{4} \leq \ldots$ so that the total discount for a package of size $n \geq 3$ is $1+\sum_{i=3}^{n} \delta_{i}$ if the package contains $A$ and $2+\sum_{i=3}^{n} \delta_{i}$ otherwise.

\footnotetext{
${ }^{37}$ An analogous counterexample can be constructed for our seller Lloyd. If Lloyd can produce object $C$ at a cost of 4 , packages $A C$ and $B C$ at a cost of 7 , and package $A B C$ at a cost of 12 , he has substitutes payoffs but is not decomposable.

${ }^{38}$ Similarly, if Lloyd's production costs for $C, A C, B C$, and $A B C$ are 4, 7, 9, and 13, then he is decomposable but his cost function does not exhibit SDD.
} 


\section{Conclusion}

Private information has been shown to be a transaction cost that prevents efficient reallocation in two-sided setups with bilateral trade or homogeneous goods. We derive conditions under which the impossibility of efficient trade extends to richer environments in which buyers and sellers have multi-dimensional types that are private information.

We begin by synthesizing the literature and showing that a result due to Shapley (1962), which implies that buyers and sellers are complements in assignment games, can be used together with the insights from the literature on dominant strategy mechanisms to prove the impossibility of efficient trades in all two-sided allocation problems with one-to-one trading. Generalizing Shapley's result to sets of unit constituents of decomposable buyers and sellers, we then provide a new impossibility theorem based on decomposability that applies to general setups in which buyers consume packages of objects produced by different sellers and sellers produce packages of objects consumed by different buyers.

Besides impossibility, decomposability implies that the efficient allocation of the two-sided allocation problem corresponds to the solution to an assignment game between objects and unit constituents, which makes the problem computationally tractable because the solution can be found using linear programming. It also implies that the core is nonempty and that competitive equilibrium prices exist (Shapley and Shubik, 1972).

Because decomposability is a condition on payoff functions, the condition for our impossibility result can be verified without having to account for the interactions between different agents, whose payoff functions may vary considerably. Furthermore, we introduce a general class of payoff functions - those with size-dependent discounts - that ensures decomposability and thus impossibility. The SDD family nests a wide range of payoff functions as special cases, including homogeneous goods, additive payoffs, unit demands and supplies, and an additively separable version of the heterogeneous commodities model of Ausubel (2006). In light of this, the classic impossibility results of Vickrey (1961), Myerson and Satterthwaite (1983), and Gresik and Satterthwaite (1989) as well as the impossibility of efficient trade for the Shapley-Shubik model arise because agents' payoff functions exhibit SDD.

This paper opens up a number of avenues for future research. Although the paper is immediately motivated by the Coase Theorem and by improving our understanding of secondary markets, and thereby relates to market design in two-sided settings (Loertscher et al., 2015; Milgrom, 2017), 
the concept of SDD payoff functions that emerges from our analysis seems of independent interest. Without additional structure, package problems suffer from a curse of dimensionality that makes evaluating packages and communicating information practically impossible. For example, even when there are only ten objects to be allocated, an agent would have to evaluate and express valuations for more than a thousand different packages. In contrast, with SDD preferences, agents would only have to evaluate and communicate the ten stand-alone utilities and nine discounts. Allowing for the expression of SDD payoffs and encoding them in assignment game form thus substantially reduces the burden for agents and the designer with regard to communication, computation, and information acquisition. From a theoretical perspective, deriving more general conditions for agents to be decomposable would seem valuable.

The general impossibility theorem we derive raises another, fundamental question: What are detail-free mechanisms that endow agents with dominant strategies, allocate close to efficiently, and never run a deficit? For homogeneous goods with unit demands and supplies, McAfee (1992) develops such a mechanism, which sacrifices at most the least valuable of all the trades that should occur under efficiency. While the extension to buyers and sellers with multi-unit demands and supplies and multi-dimensional types poses major challenges (Loertscher and Mezzetti, 2017), the monotonicity properties of the homogeneous goods model give it considerable tractability. With heterogeneous objects, much of this structure is lost. For example, in a Shapley-Shubik model, efficiency may dictate that a high cost seller trade while a low cost seller remain inactive. Progress in the development of practical, deficit-free mechanisms for heterogeneous objects is therefore likely to face formidable obstacles, yet commensurate rewards. 


\section{References}

Aizerman, M. A. And A. V. Malishevski (1981): "General Theory of Best Variants Choice: Some Aspects," IEEE Transactions on Automatic Control, 26, 1030-1040.

Arrow, K. (1959): "Toward a Theory of Price Adjustment," in The Allocation of Economic Resources: Essays in Honor of Bernard Francis Haley, ed. by M. Abramovitz, Stanford: Stanford University Press, 41-51.

Ausubel, L. (2006): "An Efficient Dynamic Auction for Heterogeneous Commodities," American Economic Review, 96, 602-629.

Bikhchandani, S. And J. M. Ostroy (2002): "The Package Assignment Model," Journal of Economic Theory, 107, 377-406.

Chambers, C. And F. Echenique (2015): "The Core Matchings of Markets with Transfers," American Economic Journal: Microeconomics, 7, 144-164.

Chambers, C. P. And M. B. Yenmez (2017): "Choice and Matching," American Economic Journal: Microeconomics, 9, 126-147.

Chen, P., M. Egesdal, M. Pycia, And M. B. Yenmez (2014): "Quantile Stable Mechanisms," Working Paper, University of Chicago.

Choo, E. (2015): "Dynamic Marriage Matching: An Empirical Framework," Econometrica, 83, $1373-1423$.

Clarke, E. (1971): "Multipart Pricing of Public Goods," Public Choice, 11, 17-33.

Cramton, P., R. Gibbons, and P. Klemperer (1987): "Dissolving a Partnership Efficiently," Econometrica, 55, 615-632.

Dantzig, G. B. (1963): Linear Programming and Extensions, Princeton University Press, Princeton.

Echenique, F., S. Lee, M. Shum, And M. B. Yenmez (2013): "The Revealed Preference Theory of Stable and Extremal Stable Matchings," Econometrica, 81, 153-71.

Echenique, F. And J. Oviedo (2006): "A Theory of Stability in Many-to-Many Matching Markets," Theoretical Economics, 1, 233-273.

Fieseler, K., T. Kittsteiner, and B. Moldovanu (2003): "Partnerships, Lemons, and Efficient Trade," Journal of Economic Theory, 113, 223-234.

Garratt, R. And M. Pycia (2016): "Efficient Bilateral Trade," Working Paper.

Green, J. And J.-J. Laffont (1977): "Characterization of Satisfactory Mechanisms for the 
Revelation of Preferences for Public Goods," Econometrica, 45, 427-438.

Gresik, T. and M. Satterthwaite (1989): "The Rate at which a Simple Market Converges to Efficiency as the Number of Traders Increases: An Asymptotic Result for Optimal Trading Mechanisms," Journal of Economic Theory, 48, 304-332.

Groves, T. (1973): "Incentives in Teams," Econometrica, 41, 617-631.

Groves, T. And M. Loeb (1975): "Incentives and Public Inputs," Journal of Public Economics, $4,211-226$.

Hatfield, J., N. ImmorlicA, And S. Kominers (2012): "Testing Substitutability," Games and Economic Behaviour, 75, 639-645.

Hatfield, J., S. Kominers, A. Nichifor, M. Ostrovsky, and A. Westkamp (2017): "Full Substitutability," Working Paper, Stanford University.

Hatfield, J. And P. Milgrom (2005): "Matching with Contracts," American Economic Review, 95, 913-935.

Holmström, B. (1979): "Groves' Scheme on Restricted Domains," Econometrica, 47, 1137-1144.

Hurwicz, L. (1972): "On Informationally Decentralized Systems," in Decision and Organization, ed. by C. B. McGuire and R. Radner, Amsterdam: North-Holland, 297-336.

(1973): "The Design of Mechanisms for Resource Allocation," American Economic Review, $63,1-30$.

Jackson, M., H. Sonnenschein, And Y. Xing (2015): "The Efficiency of Bargaining with Many Items," Working Paper, University of Chicago.

Jehiel, P. And B. Moldovanu (2001): "Efficient Design with Interdependent Valuations," Econometrica, 69, 1237-1259.

Koopmans, T. C. And M. Beckmann (1957): "Assignment Problems and the Location of Economic Activities," Econometrica, 25, 53-76.

Krishna, V. (2002): Auction Theory, Academic Press, San Diego.

Kunn, H. W. (1955): "The Hungarian Method for the Assignment Problem," Naval Research Logistics Quarterly, 2, 83-97.

Loertscher, S., L. M. Marx, And T. Wilkening (2015): "A Long Way Coming: Designing Centralized Markets with Privately Informed Buyers and Sellers," Journal of Economic Literature, 53, 857-897.

Loertscher, S. And C. Mezzetti (2017): “A Dominant Strategy, Double Clock Auction with 
Estimation-Based Tâtonnement," Mimeo, University of Melbourne.

Makowski, L. And C. Mezzetti (1994): "Bayesian and Weakly Robust First Best Mechanisms: Characterizations," Journal of Economic Theory, 64, 500-519.

Makowski, L. And J. M. Ostroy (1987): "Vickrey-Clarke-Groves Mechanisms and Perfect Competition," Journal of Economic Theory, 42, 244-261.

Mas-Colell, A., M. D. Whinston, And J. R. Green (1995): Microeconomic Theory, New York: Oxford University Press.

McAfee, R. P. (1991): "Efficient Allocation with Continuous Quantities," Journal of Economic Theory, 53, 51-74.

— (1992): "A Dominant Strategy Double Auction," Journal of Economic Theory, 56, 434-450.

Mezzetti, C. (2004): "Mechanism Design with Interdependent Valuations: Efficiency," Econometrica, 72, 1617-1626.

Milgrom, P. (2017): Discovering Prices, New York: Columbia University Press.

Myerson, R. And M. Satterthwaite (1983): "Efficient Mechanisms for Bilateral Trading," Journal of Economic Theory, 29, 265-281.

Myerson, R. B. (1981): "Optimal Auction Design," Mathematics of Operations Research, 6, $58-73$.

Ostrovsky, M. and R. Paes Leme (2015): "Gross Substitutes and Endowed Assignment Valuations," Theoretical Economics, 10, 853-865.

Roth, A. E. (1982): "The Economics of Matching: Stability and Incentives," Mathematics of Operations Research, 7, 617-628.

Satterthwaite, M., S. R. Williams, and K. E. Zachariadis (2015): "Price Discovery Using a Double Auction," SSRN Working Paper.

Satterthwaite, M. A., S. R. Williams, and K. E. Zachariadis (2014): "Optimality Versus Practicality in Market Design: A Comparison of Two Double Auctions," Games and Economic Behaviour, 86, 248-263.

Segal, I. And M. Whinston (2016): "Property Rights and the Efficiency of Bargaining," Journal of the European Economic Association, 14, 1287-1328.

Shapley, L. (1962): "Complements and Substitutes in the Optimal Assignment Problem," Naval Research Logistics Quarterly, 9, 45-48.

Shapley, L. And M. Shubik (1972): “The Assignment Game I: The Core," International Journal 
of Game Theory, 1, 111-130.

Vickrey, W. (1961): "Counterspeculation, Auction, and Competitive Sealed Tenders," Journal of Finance, 16, 8-37.

Williams, S. R. (1999): "A Characterization of Efficient, Bayesian Incentive Compatible Mechanisms," Economic Theory, 14, 155-180.

Yenmez, M. B. (2013): "Incentive-Compatible Matching Mechanisms: Consistency with Various Stability Notions," American Economic Journal: Microeconomics, 5, 120-141.

(2015): "Incentive Compatible Market Design with Applications," International Journal of Game Theory, 44, 543-569. 


\section{Appendix: Proofs}

Proof of Lemma 1: Define for every buyer $b$,

$$
W_{-b}^{V C G}\left(\mathbf{v}_{-b}, \mathbf{c}\right):=\min _{\mathbf{v}_{b} \in V_{b}} W^{*}\left(\mathbf{v}_{b}, \mathbf{v}_{-b}, \mathbf{c}\right)
$$

Similarly, for every seller $s$, define

$$
W_{-s}^{V C G}\left(\mathbf{v}, \mathbf{c}_{-s}\right):=\min _{\mathbf{c}_{s} \in C_{s}} W^{*}\left(\mathbf{v}, \mathbf{c}_{s}, \mathbf{c}_{-s}\right)
$$

Notice that $W_{-b}^{V C G}\left(\mathbf{v}_{-b}, \mathbf{c}\right)$ and $W_{-s}^{V C G}\left(\mathbf{v}, \mathbf{c}_{-s}\right)$ are independent of, respectively, $\mathbf{v}_{b}$ and $\mathbf{c}_{s}$.

The VCG mechanism selects $x^{*}(\mathbf{v}, \mathbf{c}) \in X^{*}(\mathbf{v}, \mathbf{c})$, requires each buyer to pay a transfer payment of

$$
t_{b}^{\beta}(\mathbf{v}, \mathbf{c})=W_{-b}^{V C G}\left(\mathbf{v}_{-b}, \mathbf{c}\right)-\left(W^{*}(\mathbf{v}, \mathbf{c})-u_{b}\left(x^{*}(\mathbf{v}, \mathbf{c}), \mathbf{v}_{b}\right)\right)
$$

and pays each seller a transfer payment of

$$
t_{s}^{\sigma}(\mathbf{v}, \mathbf{c})=W^{*}(\mathbf{v}, \mathbf{c})+k_{s}\left(x^{*}(\mathbf{v}, \mathbf{c}), \mathbf{c}_{s}\right)-W_{-s}^{V C G}\left(\mathbf{v}, \mathbf{c}_{-s}\right)
$$

Because we have assumed that there exists a least efficient buyer type, $\underline{\mathbf{v}}_{b}$ is the minimizer of $W^{*}\left(\mathbf{v}_{b}, \mathbf{v}_{-b}, \mathbf{c}\right)$, and similarly $\overline{\mathbf{c}}_{s}$ is the minimizer of $W^{*}\left(\mathbf{v}, \mathbf{c}_{s}, \mathbf{c}_{-s}\right)$. Thus, in our environment, $W_{-b}^{V C G}\left(\mathbf{v}_{-b}, \mathbf{c}\right)=W_{-b, .}^{*}\left(\mathbf{v}_{-b}, \mathbf{c}\right)$ and $W_{-s}^{V C G}\left(\mathbf{v}, \mathbf{c}_{-s}\right)=W_{.,-s}^{*}\left(\mathbf{v}, \mathbf{c}_{-s}\right)$.

By the revenue equivalence arguments of Green and Laffont (1977) and Holmström (1979), the VCG mechanism is the revenue maximizing mechanism among all mechanisms that respect agents' individual rationality constraints ex post and endow them with dominant strategies. Our focus on the VCG mechanism is therefore without loss of generality.

Revenue to the mechanism when the types are $(\mathbf{v}, \mathbf{c})$, denoted $R(\mathbf{v}, \mathbf{c})$, is

$$
\begin{aligned}
R(\mathbf{v}, \mathbf{c}) & =\sum_{b \in \mathbb{B}} t_{b}^{\beta}(\mathbf{v}, \mathbf{c})-\sum_{s \in \mathbb{S}} t_{s}^{\sigma}(\mathbf{v}, \mathbf{c}) \\
& =W^{*}(\mathbf{v}, \mathbf{c})+\sum_{b \in \mathbb{B}}\left[W_{-b, .}^{*}\left(\mathbf{v}_{-b}, \mathbf{c}\right)-W^{*}(\mathbf{v}, \mathbf{c})\right]+\sum_{s \in \mathbb{S}}\left[W_{.,-s}^{*}\left(\mathbf{v}, \mathbf{c}_{-s}\right)-W^{*}(\mathbf{v}, \mathbf{c})\right] .
\end{aligned}
$$

Noting that $W_{-b, .}^{*}(\mathbf{v}, \mathbf{c})$ does not depend on $\mathbf{v}_{b}$ and so $W_{-b, .}^{*}(\mathbf{v}, \mathbf{c})=W_{-b, .}^{*}\left(\mathbf{v}_{-b}, \mathbf{c}\right)$, and similarly $W_{.,-s}^{*}(\mathbf{v}, \mathbf{c})=W_{.,-s}\left(\mathbf{v}, \mathbf{c}_{-s}\right)$, the condition for VCG revenue, $R(\mathbf{v}, \mathbf{c})$, to be less than or equal to zero is equivalent to

$$
\sum_{b \in \mathbb{B}}\left[W^{*}(\mathbf{v}, \mathbf{c})-W_{-b, .}^{*}(\mathbf{v}, \mathbf{c})\right]+\sum_{s \in \mathbb{S}}\left[W^{*}(\mathbf{v}, \mathbf{c})-W_{.,-s}^{*}(\mathbf{v}, \mathbf{c})\right] \geq W^{*}(\mathbf{v}, \mathbf{c})
$$


which is equivalent to condition (2).

It is clear from the argument above that for a given $(\mathbf{v}, \mathbf{c})$, if (2) holds strictly (and so (12) holds strictly), then VCG revenue is negative, i.e., $R(\mathbf{v}, \mathbf{c})<0$.

Proof of Lemma 3: Consider the allocation problem without buyers in $\mathbb{I}$ and sellers in $\mathbb{J}$. Let $x$ be any allocation in that problem and let $x^{0}$ be the empty allocation (i.e., $x_{b}^{0}=\varnothing$ for all $b \in \mathbb{B} \backslash \mathbb{I}$ and $x_{s}^{0}=\varnothing$ for all $\left.s \in \mathbb{S} \backslash \mathbb{J}\right)$. Let $L^{x}$ and $L^{x^{0}}$ be best isomorphic matchings for these two allocations in $A_{-\hat{\mathbb{I}} \cup \hat{\mathbb{J}},-\mathbb{O}_{\mathfrak{J}}}$. By Definition 2,

$$
\begin{aligned}
& u_{b}\left(x_{b}, \mathbf{v}_{b}\right)=\sum_{r \in \hat{\mathbb{B}}_{b}} \sum_{o \in \mathbb{O} \backslash \mathbb{O}_{\mathbb{J}}} l_{r, o}^{x} a_{r, o} \quad \text { for all } b \in \mathbb{B} \backslash \mathbb{I} \text {, and } \\
& k_{s}\left(x_{s}, \mathbf{c}_{s}\right)=\sum_{r \in \hat{\mathbb{S}}_{s}} \sum_{o \in \mathbb{O} \backslash \mathbb{O}_{\mathbb{J}}}\left[l_{r, o}^{x^{0}} a_{r, o}-l_{r, o}^{x} a_{r, o}\right] \text { for all } s \in \mathbb{S} \backslash \mathbb{J} .
\end{aligned}
$$

Recall that social gains from trade $W_{-\mathbb{I},-\mathbb{J}}(x, \mathbf{v}, \mathbf{c})$ is

$$
W_{-\mathbb{I},-\mathbb{I}}(x, \mathbf{v}, \mathbf{c})=\sum_{b \in \mathbb{B} \backslash \mathbb{I}} u_{b}\left(x_{b}, \mathbf{v}_{b}\right)-\sum_{s \in \mathbb{S} \backslash \mathbb{I}} k_{s}\left(x_{s}, \mathbf{c}_{s}\right) .
$$

Combining these two results yields

$$
\begin{aligned}
W_{-\mathbb{I},-\mathbb{J}}(x, \mathbf{v}, \mathbf{c}) & =\sum_{b \in \mathbb{B} \backslash \mathbb{I}} \sum_{r \in \hat{\mathbb{B}}_{b}} \sum_{o \in \mathbb{O} \backslash \mathbb{O}_{\mathbb{J}}} l_{r, o}^{x} a_{r, o}-\sum_{s \in \mathbb{S} \backslash \mathbb{J}} \sum_{r \in \hat{\mathbb{S}}_{s}} \sum_{o \in \mathbb{O} \backslash \mathbb{O}_{\mathbb{J}}}\left[l_{r, o}^{x^{0}} a_{r, o}-l_{r, o}^{x} a_{r, o}\right] \\
& =\sum_{r \in(\hat{\mathbb{B}} \backslash \hat{\mathbb{I}}) \cup(\hat{\mathbb{S}} \backslash \hat{\mathbb{J}})} \sum_{o \in \mathbb{O} \backslash \mathbb{O}_{\mathbb{J}}} l_{r, o}^{x} a_{r, o}-\sum_{r \in \hat{\mathbb{S}} \backslash \hat{\mathbb{J}}} \sum_{o \in \mathbb{O} \backslash \mathbb{O}_{\mathbb{J}}} l_{r, o}^{x^{0}} a_{r, o} .
\end{aligned}
$$

Recalling that $L^{0}$ optimally matches the unit constituents of each seller $s$ with the objects in $\mathbb{O}_{s}$,

$$
\sum_{r \in \hat{\mathbb{S}} \backslash \hat{\mathbb{J}}} \sum_{o \in \mathbb{O} \backslash \mathbb{O}_{\mathbb{J}}} l_{r, o}^{x^{0}} a_{r, o}=V^{*}\left(A_{-\hat{\mathbb{B}} \cup \hat{\mathbb{J}},-\mathbb{I}_{\mathbb{J}}}\right) \quad \text { for any } \mathbb{J} \subseteq \mathbb{S} .
$$

Substituting this into the equation above yields

$$
W_{-\mathbb{I},-\mathbb{J}}(x, \mathbf{v}, \mathbf{c})=\sum_{r \in(\hat{\mathbb{B}} \backslash \hat{\mathbb{I}}) \cup(\hat{\mathbb{S}} \backslash \hat{\mathbb{J}})} \sum_{o \in \mathbb{O} \backslash \mathbb{O}_{\mathbb{J}}} l_{r, o}^{x} a_{r, o}-V^{*}\left(A_{-\hat{\mathbb{B}} \cup \hat{\mathbb{J}},-\mathbb{O}_{\mathbb{J}}}\right) .
$$

To complete the proof, it remains to show that $L^{x^{*}}$ is an optimal matching of $A_{-\hat{\mathbb{I}} \cup \hat{\mathbb{J}},-\mathbb{O}_{\mathrm{J}}}$, where $x^{*}$ is a feasible maximizer of $W_{-\mathbb{I},-\mathbb{J}}(x, \mathbf{v}, \mathbf{c})$ and $L^{x^{*}}$ the best isomorphic matching associated with $x^{*}$. To see this, consider any feasible matching of $A_{-\hat{\mathbb{I}} \cup \hat{J},-\mathbb{O}_{\mathfrak{J}}}$. Because it assigns each object at most once, it is an isomorphic matching of some allocation. The output it produces is therefore at most the output produced by a best isomorphic matching of that allocation. By 
(13) and because $x^{*}$ is efficient, this cannot exceed the output created by $L^{x^{*}}$, which is equal to $W_{-\mathbb{I},-\mathbb{J}}^{*}+V^{*}\left(A_{-\hat{\mathbb{B}} \cup \hat{\mathbb{J}},-\mathbb{O}_{\mathbb{J}}}\right)$. The latter is therefore an optimal matching of $A_{-\hat{\mathbb{I}} \cup \hat{\mathbb{J}},-\mathbb{O}_{\mathbb{J}}}$. Consequently, $\sum_{r \in(\hat{\mathbb{B}} \backslash \hat{\mathbb{I}}) \cup(\hat{\mathbb{S}} \backslash \hat{\mathbb{J}})} \sum_{o \in \mathbb{O}} l_{r, o}^{x^{*}} a_{r, o}=V^{*}\left(A_{-\hat{\mathbb{I}} \cup \hat{\mathbb{J}},-\mathbb{O}_{\mathbb{J}}}\right)$ and

$$
W_{-\mathbb{I},-\mathbb{J}}^{*}=V^{*}\left(A_{-\hat{\mathbb{I}} \cup \hat{\mathbb{J}},-\mathbb{O}_{\mathbb{J}}}\right)-V^{*}\left(A_{-\hat{\mathbb{B}} \cup \hat{\mathbb{J}},-\mathbb{O}_{\mathbb{J}}}\right) .
$$

Proof of Lemma 4: We show that (2) and (8) are equivalent.

Taking $\mathbb{I}=\{b\}$ and $\mathbb{J}=\varnothing$ in equation (5) of Lemma 3 gives us $W_{-b, .}^{*}=V^{*}\left(A_{-\hat{\mathbb{B}}_{b}, .}\right)-V^{*}\left(A_{-\hat{\mathbb{B}}_{,} .}\right)$, and taking $\mathbb{I}=\varnothing$ and $\mathbb{J}=\varnothing$ gives $W^{*}=V^{*}(A)-V^{*}\left(A_{-\hat{\mathbb{B}}, .}\right)$. Thus, summing over all $b$ in $\mathbb{B}$,

$$
\sum_{b \in \mathbb{B}}\left[W^{*}-W_{-b, .}^{*}\right]=\sum_{b \in \mathbb{B}}\left[V^{*}(A)-V^{*}\left(A_{-\hat{\mathbb{B}}_{b}, .}\right)\right] .
$$

Taking $\mathbb{I}=\varnothing$ and $\mathbb{J}=\{s\}$ in (5) implies that $W_{.,-s}^{*}=V^{*}\left(A_{-\hat{\mathbb{S}}_{s},-\mathbb{O}_{s}}\right)-V^{*}\left(A_{-\hat{\mathbb{B}}_{\cup} \hat{\mathbb{S}}_{s},-\mathbb{O}_{s}}\right)$. Thus,

$$
W^{*}-W_{.,-s}^{*}=\left[V^{*}(A)-V^{*}\left(A_{-\hat{\mathbb{S}}_{s},-\mathbb{O}_{s}}\right)\right]-\left[V^{*}\left(A_{-\hat{\mathbb{B}}_{,}}\right)-V^{*}\left(A_{-\hat{\mathbb{B}}_{\cup} \cup \hat{\mathbb{S}}_{s},-\mathbb{O}_{s}}\right)\right] .
$$

Summing over all $s \in \mathbb{S}$ and noting that the final term in the expression above is the value of optimally assigning all objects in $\mathbb{O}_{s}$ to unit constituents in $\hat{\mathbb{S}}_{s}$, which when summed over all sellers gives $V^{*}\left(A_{-\hat{\mathbb{B}}, .}\right)-V^{*}\left(A_{-\hat{\mathbb{B}} \cup \hat{\mathbb{S}},-\mathbb{O}}\right)=V^{*}\left(A_{-\hat{\mathbb{B}}, .}\right)$, we have

$$
\sum_{s \in \mathbb{S}}\left[W^{*}-W_{.,-s}^{*}\right]=\sum_{s \in \mathbb{S}}\left[V^{*}(A)-V^{*}\left(A_{-\hat{\mathbb{S}}_{s},-\mathbb{O}_{s}}\right)\right]-V^{*}\left(A_{-\hat{\mathbb{B}}, .}\right) .
$$

Recall from above that

$$
W^{*}=V^{*}(A)-V^{*}\left(A_{-\hat{\mathbb{B}}, .}\right) .
$$

Combining (14), (15), and (16), and noting that the final terms in (15) and (16) are the same, it follows that (2) holds in an assignment-representable problem if and only if (8) holds, and holds strictly if and only if (8) holds strictly.

Proof of Lemma 5: We begin by proving part (ii), showing that (10) holds. Observe first that (10) holds with an equality if $\hat{T} \leq 1$, so we assume that $\hat{T} \geq 2$. Label the elements of $\hat{\mathbb{T}}$ such that $\hat{\mathbb{T}}=\left\{r_{1}, \ldots, r_{\hat{T}}\right\}$ and define $\hat{\mathbb{T}}_{n}:=\left\{r_{1}, \ldots, r_{n}\right\}$ for any $n \in\{1, \ldots, \hat{T}\}$ as well as $\hat{\mathbb{T}}_{0}:=\varnothing$. By Lemma 2 , for any $m \in\{2, \ldots, \hat{T}\}$ and $n \in\{1, \ldots, m-1\}, r_{m}$ and $r_{n}$ are substitutes for each other in the assignment game with value matrix $A_{-\hat{\mathbb{T}}_{n-1},}$. That is, for any $m \in\{2, \ldots, \hat{T}\}$ and $n \in\{1, \ldots, m-1\}$, $V^{*}\left(A_{-\hat{\mathbb{T}}_{n-1}, .}\right)-V^{*}\left(A_{-\hat{\mathbb{T}}_{n-1} \cup\left\{r_{m}\right\}, .}\right)+V^{*}\left(A_{-\hat{\mathbb{T}}_{n-1}, .}\right)-V^{*}\left(A_{-\hat{\mathbb{T}}_{n}, .}\right) \leq V^{*}\left(A_{-\hat{\mathbb{T}}_{n-1}, .}\right)-V^{*}\left(A_{-\hat{\mathbb{T}}_{n} \cup\left\{r_{m}\right\}, .}\right)$, 
which can be rearranged as

$$
V^{*}\left(A_{-\hat{\mathbb{T}}_{n-1}, .}\right)-V^{*}\left(A_{-\hat{\mathbb{T}}_{n-1} \cup\left\{r_{m}\right\}, .}\right) \leq V^{*}\left(A_{-\hat{\mathbb{T}}_{n}, .}\right)-V^{*}\left(A_{-\hat{\mathbb{T}}_{n} \cup\left\{r_{m}\right\}, .}\right) .
$$

Evaluating (17) at $n=1, \ldots, m-1$ gives us a series of $m-1$ inequalities of the form

$$
\begin{aligned}
V^{*}(A)-V^{*}\left(A_{-r_{m}, .}\right) & \leq V^{*}\left(A_{-r_{1}, .}\right)-V^{*}\left(A_{-\left\{r_{1}, r_{m}\right\}, .}\right) \\
V^{*}\left(A_{-r_{1}, .}\right)-V^{*}\left(A_{-\left\{r_{1}, r_{m}\right\}, .}\right) & \leq V^{*}\left(A_{-\left\{r_{1}, r_{2}\right\}, .}\right)-V^{*}\left(A_{-\left\{r_{1}, r_{2}, r_{m}\right\}, .}\right) \\
& \ldots \\
V^{*}\left(A_{-\hat{\mathbb{T}}_{m-2,} .}\right)-V^{*}\left(A_{-\hat{\mathbb{T}}_{m-2} \cup\left\{r_{m}\right\}, .}\right) & \leq V^{*}\left(A_{-\hat{\mathbb{T}}_{m-1, .}}\right)-V^{*}\left(A_{-\hat{\mathbb{T}}_{m}, .}\right),
\end{aligned}
$$

which implies

$$
V^{*}(A)-V^{*}\left(A_{-r_{m}, .}\right) \leq V^{*}\left(A_{-\hat{\mathbb{T}}_{m-1}, .}\right)-V^{*}\left(A_{-\hat{\mathbb{T}}_{m}, .}\right) .
$$

Because (17) holds for any $m \in\{2, \ldots, \hat{T}\}$, so does (18). When $m=1, \hat{\mathbb{T}}_{m-1}=\hat{\mathbb{T}}_{0}:=\varnothing$, in which case (18) is satisfied with an equality. Therefore (18) implies

$$
\begin{aligned}
\sum_{m=1}^{\hat{T}}\left[V^{*}(A)-V^{*}\left(A_{-r_{m}, .}\right)\right] & \leq \sum_{m=1}^{\hat{T}}\left[V^{*}\left(A_{-\hat{\mathbb{T}}_{m-1} . .}\right)-V^{*}\left(A_{-\hat{\mathbb{T}}_{m}, .}\right)\right] \\
& =V^{*}\left(A_{-\hat{\mathbb{T}}_{0} .}\right)-V^{*}\left(A_{-\hat{\mathbb{T}}_{\hat{T}}, .}\right) \\
& =V^{*}(A)-V^{*}\left(A_{-\hat{\mathbb{T}}, .}\right),
\end{aligned}
$$

where the first equality expands the sum and cancels terms and the final equality uses $\hat{\mathbb{T}}_{0}=\varnothing$ and $\hat{\mathbb{T}}_{\hat{T}}=\hat{\mathbb{T}}$. This completes the proof that (10) holds and so completes the proof of part (ii).

We now turn to the proof of part (i), showing that (9) holds. Similar to the case above, (9) holds with an equality if either $\hat{\mathbb{T}}=\varnothing$ or $\hat{\mathbb{T}}^{\prime}=\varnothing$, so we focus on the case in which both subsets contain at least one element. As before, let $\hat{\mathbb{T}}_{n}:=\left\{r_{1}, \ldots, r_{n}\right\}$ for any $n \in\{1, \ldots, \hat{T}\}$ and, analogously, let $\hat{\mathbb{T}}_{m}^{\prime}:=\left\{r_{1}^{\prime}, \ldots, r_{m}^{\prime}\right\}$ for any $m \in\left\{1, \ldots, \hat{T}^{\prime}\right\}$. Define $\hat{\mathbb{T}}_{0}:=\varnothing$ and $\hat{\mathbb{T}}_{0}^{\prime}:=\varnothing$.

By Lemma 2, for any $n \in\{1, \ldots, \hat{T}\}$ and $m \in\left\{1, \ldots, \hat{T}^{\prime}\right\}, r_{n}$ and $r_{m}^{\prime}$ are substitutes for each other in the assignment game with value matrix $A_{-\hat{\mathbb{T}}_{n-1} \cup \hat{\mathbb{T}}_{m-1}^{\prime}, .}$. That is, for any $n \in\{1, \ldots, \hat{T}\}$ and $m \in\left\{1, \ldots, \hat{T}^{\prime}\right\}$,

$$
\begin{aligned}
V^{*}\left(A_{-\hat{\mathbb{T}}_{n-1} \cup \hat{\mathbb{T}}_{m-1}^{\prime}, .}\right)-V^{*}\left(A_{-\hat{\mathbb{T}}_{n} \cup \hat{\mathbb{T}}_{m-1}^{\prime}, .}\right) & +V^{*}\left(A_{-\hat{\mathbb{T}}_{n-1} \cup \hat{\mathbb{T}}_{m-1}^{\prime}, .}\right)-V^{*}\left(A_{-\hat{\mathbb{T}}_{n-1} \cup \hat{\mathbb{T}}_{m}^{\prime}, .}\right) \\
& \leq V^{*}\left(A_{-\hat{\mathbb{T}}_{n-1} \cup \hat{\mathbb{T}}_{m-1}^{\prime}, .}\right)-V^{*}\left(A_{-\hat{\mathbb{T}}_{n} \cup \hat{\mathbb{T}}_{m}^{\prime}, .}\right) .
\end{aligned}
$$

By rearranging the above inequality, we obtain

$$
V^{*}\left(A_{-\hat{\mathbb{T}}_{n-1} \cup \hat{\mathbb{T}}_{m-1}^{\prime}, .}\right)-V^{*}\left(A_{-\hat{\mathbb{T}}_{n} \cup \hat{\mathbb{T}}_{m-1}^{\prime}, .}\right) \leq V^{*}\left(A_{-\hat{\mathbb{T}}_{n-1} \cup \hat{\mathbb{T}}_{m}^{\prime}, .}\right)-V^{*}\left(A_{-\hat{\mathbb{T}}_{n} \cup \hat{\mathbb{T}}_{m}^{\prime}, .}\right) .
$$


By an analogous argument to the one developed in the proof of part (ii), keeping $n$ fixed and invoking (19) for each possible value of $m$ between 1 and $\hat{T}^{\prime}$ yields a series of $\hat{T}^{\prime}$ inequalities such that the right side of each is equal to the left side of the next. The left side of the first one must therefore be weakly smaller than the right side of the last one, that is,

$$
V^{*}\left(A_{-\hat{\mathbb{T}}_{n-1}, .}\right)-V^{*}\left(A_{-\hat{\mathbb{T}}_{n}, .}\right) \leq V^{*}\left(A_{-\hat{\mathbb{T}}_{n-1} \cup \hat{\mathbb{T}}^{\prime}, .}\right)-V^{*}\left(A_{-\hat{\mathbb{T}}_{n} \cup \hat{\mathbb{T}}^{\prime}, .}\right),
$$

which is equivalent to

$$
V^{*}\left(A_{-\hat{\mathbb{T}}_{n-1}, .}\right)-V^{*}\left(A_{-\hat{\mathbb{T}}_{n-1} \cup \hat{\mathbb{T}}^{\prime}, .}\right) \leq V^{*}\left(A_{-\hat{\mathbb{T}}_{n}, .}\right)-V^{*}\left(A_{-\hat{\mathbb{T}}_{n} \cup \hat{\mathbb{T}}^{\prime},}\right) .
$$

Because this inequality holds for any $n \in\{1, \ldots, \hat{T}\}$, analogous reasoning to above yields

$$
V^{*}(A)-V^{*}\left(A_{-\hat{\mathbb{T}}^{\prime}, .}\right) \leq V^{*}\left(A_{-\hat{\mathbb{T}}, .}\right)-V^{*}\left(A_{-\hat{\mathbb{T}} \cup \hat{\mathbb{T}}^{\prime}, .}\right)
$$

which is equivalent to (9) and completes the proof of part (i).

By the symmetry of assignment games, the proofs of parts (iii) and (iv) are analogous.

We conclude with the proof of part (v), showing that (11) holds. Similarly to the proofs of parts (i) and (ii), (11) holds with an equality if either $\hat{\mathbb{T}}=\varnothing$ or $\mathbb{K}=\varnothing$, hence we focus on the case in which both subsets contain at least one element. As before, let $\hat{\mathbb{T}}_{n}:=\left\{r_{1}, \ldots, r_{n}\right\}$ for any $n \in\{1, \ldots, \hat{T}\}$ and, analogously, let $\mathbb{K}_{m}:=\left\{o_{1}, \ldots, o_{m}\right\}$ for any $m \in\{1, \ldots, K\}$. Define $\hat{\mathbb{T}}_{0}:=\varnothing$ and $\mathbb{K}_{0}:=\varnothing$.

By Lemma 2, for any $n \in\{1, \ldots, \hat{T}\}$ and $m \in\{1, \ldots, K\}, r_{n}$ and $o_{m}$ are complements to each other in the assignment game with value matrix $A_{-\hat{\mathbb{T}}_{n-1},-\mathbb{K}_{m-1}}$. That is, for any $n \in\{1, \ldots, \hat{T}\}$ and $m \in\{1, \ldots, K\}$,

$$
\begin{aligned}
V^{*}\left(A_{-\hat{\mathbb{T}}_{n-1},-\mathbb{K}_{m-1}}\right)-V^{*}\left(A_{-\hat{\mathbb{T}}_{n},-\mathbb{K}_{m-1}}\right) & +V^{*}\left(A_{-\hat{\mathbb{T}}_{n-1},-\mathbb{K}_{m-1}}\right)-V^{*}\left(A_{-\hat{\mathbb{T}}_{n-1},-\mathbb{K}_{m}}\right) \\
& \leq V^{*}\left(A_{-\hat{\mathbb{T}}_{n-1},-\mathbb{K}_{m-1}}\right)-V^{*}\left(A_{-\hat{\mathbb{T}}_{n},-\mathbb{K}_{m}}\right) .
\end{aligned}
$$

By rearranging the above inequality, we obtain

$$
V^{*}\left(A_{-\hat{\mathbb{T}}_{n-1},-\mathbb{K}_{m-1}}\right)-V^{*}\left(A_{-\hat{\mathbb{T}}_{n},-\mathbb{K}_{m-1}}\right) \leq V^{*}\left(A_{-\hat{\mathbb{T}}_{n-1},-\mathbb{K}_{m}}\right)-V^{*}\left(A_{-\hat{\mathbb{T}}_{n},-\mathbb{K}_{m}}\right) .
$$

The remainder of the proof of part (v) is analogous to the proof of part (ii) starting after (17).

Proof of Theorem 1: Lemma 4 establishes that (8) implies (2), and the example given prior to Proposition 1 shows that it is satisfied strictly for some types. Thus, it remains to show that (8) holds for any unit constituent-object assignment game with value matrix $A$. 
Let $L^{*}$ be an optimal matching of $A$ such that

(i) $\sum_{r \in \hat{\mathbb{B}} \cup \hat{\mathbb{S}}} l_{r, o}^{*}=1$ for all $o \in \mathbb{O}$, and

(ii) $l_{r, o}^{*}=0$ for all pairs $(r, o)$ such that $r \in \hat{\mathbb{S}}_{s}$ and $o \in \mathbb{O}_{s^{\prime}}$ where $s, s^{\prime} \in \mathbb{S}$ with $s \neq s^{\prime}$.

An optimal matching satisfying (i) and (ii) always exists: An optimal matching satisfying (i), which states no object is left unmatched, always exists because objects are on the short side and the elements of $A$ are nonnegative. Moreover, by Definition $1, a_{r, o}=0$ if $r \in \hat{\mathbb{S}}_{s}$ and $o \in \mathbb{O}_{s^{\prime}}$ with $s \neq s^{\prime}$. Consequently, one can always find an optimal matching that only matches seller unit constituents with objects that belong to their seller's potential set, as required by (ii).

For all $o \in \mathbb{O}$, let $s(o) \in \mathbb{S}$ be the seller that is able to produce object $o$ (i.e., $o \in \mathbb{O}_{s(o)}$ ), and let $r^{*}(o) \in \hat{\mathbb{B}} \cup\{s(o)\}$ be the unit constituent that $L^{*}$ matches with object $o$. Then $l_{r^{*}(o), o}^{*}=1$ for all $o \in \mathbb{O}$ and $l_{r, o}^{*}=0$ for all $(r, o)$ with $r \neq r^{*}(o)$. It follows that

$$
V^{*}(A)=\sum_{r \in \hat{\mathbb{B}} \cup \hat{\mathbb{S}}} \sum_{o \in \mathbb{O}} l_{r, o}^{*} a_{r, o}=\sum_{o \in \mathbb{O}} a_{r^{*}(o), o} .
$$

Let $\widetilde{\mathbb{O}}_{s}:=\left\{o \in \mathbb{O}_{s} \mid r^{*}(o) \in \hat{\mathbb{B}}\right\}$ be the subset of objects in seller $s$ 's potential set that are matched to a buyer unit constituent. Then $\mathbb{O}_{s} \backslash \widetilde{\mathbb{O}}_{s}$ represents the set of objects matched to unit constituents of $s$. Let $\widetilde{\mathbb{O}}:=\cup_{s \in \mathbb{S}} \widetilde{\mathbb{O}}_{s}=\left\{o \in \mathbb{O} \mid r^{*}(o) \in \hat{\mathbb{B}}\right\}$ be the set of objects matched to a unit constituent of a buyer.

Lemma 2 implies that the marginal product of each unit constituent and each object exceeds the marginal product generated by the pair. This implies that:

$$
\sum_{o \in \widetilde{\mathbb{O}}}\left[V^{*}(A)-V^{*}\left(A_{-r^{*}(o), .}\right)+V^{*}(A)-V^{*}\left(A_{.,-o}\right)\right] \geq \sum_{o \in \widetilde{\mathbb{O}}}\left[V^{*}(A)-V^{*}\left(A_{-r^{*}(o),-o}\right)\right] .
$$

Because $o$ and $r^{*}(o)$ are optimally matched together, removing that pair does not affect the rest of the assignment game. Consequently, its marginal product is $V^{*}(A)-V^{*}\left(A_{-r^{*}(o),-o}\right)=a_{r^{*}(o), o}$, and the right side of $(20)$ is equal to $\sum_{o \in \widetilde{\mathbb{O}}} a_{r^{*}(o), o}$.

Notice next that

$$
\sum_{o \in \widetilde{\mathbb{O}}}\left[V^{*}(A)-V^{*}\left(A_{-r^{*}(o), .}\right)\right]=\sum_{\hat{b} \in \hat{\mathbb{B}}}\left[V^{*}(A)-V^{*}\left(A_{-\hat{b}, .}\right)\right]
$$

because each object in $\widetilde{\mathbb{O}}$ is matched to a buyer unit constituent and each one of the latter is either unmatched or matched to an object in $\widetilde{\mathbb{O}}$. By part (ii) of Lemma 5 , for any $b \in \mathbb{B}$ we have

$$
V^{*}(A)-V^{*}\left(A_{-\hat{\mathbb{B}}_{b}, .}\right) \geq \sum_{\hat{b} \in \hat{\mathbb{B}}_{b}}\left[V^{*}(A)-V^{*}\left(A_{-\hat{b}, .}\right)\right]
$$


while part (iv) of Lemma 5 implies for any $s \in \mathbb{S}$

$$
V^{*}(A)-V^{*}\left(A_{.,-, \widetilde{\mathbb{O}}_{s}}\right) \geq \sum_{\hat{o} \in \tilde{\mathbb{O}}_{s}}\left[V^{*}(A)-V^{*}\left(A_{.,-o}\right)\right]
$$

Adding up these terms over the buyers and sellers yields

$$
\begin{aligned}
\sum_{b \in \mathbb{B}}\left[V^{*}(A)-V^{*}\left(A_{-\hat{\mathbb{B}}_{b}, .}\right)\right] & \geq \sum_{\hat{b} \in \hat{\mathbb{B}}}\left[V^{*}(A)-V^{*}\left(A_{-\hat{b}, .}\right)\right] \\
\text { and } \sum_{s \in \mathbb{S}}\left[V^{*}(A)-V^{*}\left(A_{.,-} \widetilde{\mathbb{O}}_{s}\right)\right] & \geq \sum_{o \in \widetilde{\mathbb{O}}}\left[V^{*}(A)-V^{*}\left(A_{.,-o}\right)\right] .
\end{aligned}
$$

Using these results along with (20) yields

$$
\sum_{b \in \mathbb{B}}\left[V^{*}(A)-V^{*}\left(A_{-\hat{\mathbb{B}}_{b}, .}\right)\right]+\sum_{s \in \mathbb{S}}\left[V^{*}(A)-V^{*}\left(A_{.,-\widetilde{\mathbb{O}}_{s}}\right)\right] \geq \sum_{o \in \widetilde{\mathbb{O}}} a_{r^{*}}(o), o
$$

Removing unit constituents cannot increase the value created by an assignment game, that is, $V^{*}\left(A_{.,-\widetilde{\mathbb{O}}_{s}}\right) \geq V^{*}\left(A_{-\hat{\mathbb{S}}_{s},-\widetilde{\mathbb{O}}_{s}}\right)$. Combined with $(21)$, this yields

$$
\sum_{b \in \mathbb{B}}\left[V^{*}(A)-V^{*}\left(A_{-\hat{\mathbb{B}}_{b},}\right)\right]+\sum_{s \in \mathbb{S}}\left[V^{*}(A)-V^{*}\left(A_{-\hat{\mathbb{S}}_{s},-\widetilde{\mathbb{O}}_{s}}\right)\right] \geq \sum_{o \in \widetilde{\mathbb{O}}} a_{r^{*}(o), o}
$$

We next look at the marginal product of objects in $\mathbb{O} \backslash \widetilde{\mathbb{O}}$. Each object in $\mathbb{O}_{s} \backslash \widetilde{\mathbb{O}}_{s}$ is matched to a unit constituent of $s$, and each unit constituent of $s$ is either unmatched or matched to an object in $\mathbb{O}_{s} \backslash \widetilde{\mathbb{O}}_{s}$. Therefore for any $s \in \mathbb{S}$, removing $\hat{\mathbb{S}}_{s}$ and $\mathbb{O}_{s} \backslash \widetilde{\mathbb{O}}_{s}$ implies that unmatched unit constituents and matched pairs are removed. Hence, for all $s \in \mathbb{S}$,

$$
V^{*}(A)-V^{*}\left(A_{-\hat{\mathbb{S}}_{s},-\mathbb{O}_{s} \backslash \widetilde{\mathbb{O}}_{s}}\right)=\sum_{o \in \mathbb{O}_{s} \backslash \widetilde{\mathbb{O}}_{s}} a_{r^{*}(o), o}
$$

Using this result in conjunction with (22) yields

$$
\begin{aligned}
& \sum_{b \in \mathbb{B}}\left[V^{*}(A)-V^{*}\left(A_{-\hat{\mathbb{B}}_{b}, .}\right)\right]+\sum_{s \in \mathbb{S}}\left[V^{*}(A)-V^{*}\left(A_{-\hat{\mathbb{S}}_{s},-\widetilde{\mathbb{O}}_{s}}\right)+V^{*}(A)-V^{*}\left(A_{-\hat{\mathbb{S}}_{s},-\mathbb{O}_{s} \backslash \widetilde{\mathbb{O}}_{s}}\right)\right] \\
& \geq \sum_{o \in \mathbb{O}} a_{r^{*}(o), o}
\end{aligned}
$$

We now invoke part (iii) of Lemma 5 to obtain, for all $s \in \mathbb{S}$,

$$
V^{*}(A)-V^{*}\left(A_{-\hat{\mathbb{S}}_{s},-\mathbb{O}_{s}}\right) \geq V^{*}(A)-V^{*}\left(A_{-\hat{\mathbb{S}}_{s},-\widetilde{\mathbb{O}}_{s}}\right)+V^{*}(A)-V^{*}\left(A_{-\hat{\mathbb{S}}_{s},-\mathbb{O}_{s} \backslash \widetilde{\mathbb{O}}_{s}}\right) .
$$

and thus

$$
\sum_{b \in \mathbb{B}}\left[V^{*}(A)-V^{*}\left(A_{-\hat{\mathbb{B}}_{b},}\right)\right]+\sum_{s \in \mathbb{S}}\left[V^{*}(A)-V^{*}\left(A_{-\hat{\mathbb{S}}_{s},-\mathbb{O}_{s}}\right)\right] \geq \sum_{o \in \mathbb{O}} a_{r^{*}(o), o} .
$$


Observe finally that all objects in $\mathbb{O}$ are matched to a unit constituent in $\hat{\mathbb{B}} \cup \hat{\mathbb{S}}$ and all unit constituents in $\hat{\mathbb{B}} \cup \hat{\mathbb{S}}$ are either unmatched or matched to an object in $\mathbb{O}$. Removing $\hat{\mathbb{B}} \cup \hat{\mathbb{S}}$ and $\mathbb{O}$ means removing matched pairs and unmatched unit constituents so

$$
V^{*}(A)-V^{*}\left(A_{-\hat{\mathbb{B}} \cup \hat{\mathbb{S}},-\mathbb{O}}\right)=\sum_{o \in \mathbb{O}} a_{r^{*}(o), o}
$$

Combining the last two results yields (8), which completes the proof.

Proof of Proposition 2: (If) Suppose that the allocation problem is such that all buyers and all sellers are decomposable. Then it is possible to construct a $(B O+O) \times O$ matrix $A$ such that the rows devoted to unit constituents of each agent are those of his decomposition. That is,

$$
A:=\left(\begin{array}{ccccc} 
& & A^{b_{1}} & & \\
& & A^{b_{2}} & & \\
& & A^{b_{3}} & & \\
& & \vdots & & \\
& & A^{b_{B}} & & \\
A^{s_{1}} & \mathbf{0} & \mathbf{0} & \cdots & \mathbf{0} \\
\mathbf{0} & A^{s_{2}} & \mathbf{0} & \cdots & \mathbf{0} \\
\mathbf{0} & \mathbf{0} & A^{s_{3}} & \cdots & \mathbf{0} \\
\vdots & \vdots & \vdots & \ddots & \vdots \\
\mathbf{0} & \mathbf{0} & \mathbf{0} & \cdots & A^{s_{S}}
\end{array}\right),
$$

where $A^{b_{i}}$ is the decomposition of the $i$-th buyer and $A^{s_{j}}$ the decomposition of the $j$-th seller. Observe that $A$ satisfies Definition 1 by construction. Consider a feasible allocation $x$ and recall that $\mathbb{L}(A, x)$ is the set of feasible matchings of $A$ that are isomorphic to $x$. Notice that the elements of $\mathbb{L}(A, x)$ only differ in the way they assign objects across unit constituents of the same agent. A best isomorphic matching of $x$ in $A, L^{x}$, is one that, for each buyer $b$ assigns the objects in $x_{b}$ to unit constituents in $\hat{\mathbb{B}}_{b}$ optimally and for each seller $s$ assigns the objects in $\mathbb{O}_{s} \backslash x_{s}$ to unit constituents in $\hat{\mathbb{S}}_{s}$ optimally. (If this were not the case, then another isomorphic matching would exist that produces a larger value, contradicting that $L^{x}$ is a best isomorphic matching.) This implies the first equality sign in each of the following two lines:

$$
\begin{aligned}
V^{*}\left(A_{x_{b}}^{b}\right)=\sum_{r \in \hat{\mathbb{B}}_{b}} \sum_{o \in x_{b}} l_{r, o}^{x} a_{r, o} & =\sum_{r \in \hat{\mathbb{B}}_{b}} \sum_{o \in \mathbb{O}} l_{r, o}^{x} a_{r, o} \quad \text { for all } b \in \mathbb{B} \quad \text { and } \\
V^{*}\left(A_{\mathbb{O}_{s} \backslash x_{s}}^{s}\right)=\sum_{r \in \hat{\mathbb{S}}_{s}} \sum_{o \in \mathbb{O}_{s} \backslash x_{s}} l_{r, o}^{x} a_{r, o} & =\sum_{r \in \hat{\mathbb{S}}_{s}} \sum_{o \in \mathbb{O}} l_{r, o}^{x} a_{r, o} \quad \text { for all } s \in \mathbb{S} .
\end{aligned}
$$


On both lines, the second equality sign derives from the fact that $L^{x}$ is isomorphic to $x$, which means (i) $l_{r, o}^{x}=0$ for all $r \in \hat{\mathbb{B}}_{b}$ and $o \in \mathbb{O}$ such that $o \notin x_{b}$ and (ii) $l_{r, o}^{x}=0$ for all $r \in \hat{\mathbb{S}}_{s}$ and $o \in \mathbb{O}$ such that $o \notin \mathbb{O}_{s} \backslash x_{s}$.

Because this is true for any feasible allocation, it also holds for the empty allocation $x^{0}$ and its best isomorphic matching $L^{x^{0}}$ :

$$
V^{*}\left(A^{s}\right)=\sum_{r \in \hat{\mathbb{S}}_{s}} \sum_{o \in \mathbb{O}_{s}} l_{r, o}^{x^{0}} a_{r, o}=\sum_{r \in \hat{\mathbb{S}}_{s}} \sum_{o \in \mathbb{O}} l_{r, o}^{x^{0}} a_{r, o} \quad \text { for all } s \in \mathbb{S} \text {. }
$$

Combining these results with the assumption that all agents are decomposable yields

$$
\begin{aligned}
u_{b}\left(x_{b}, \mathbf{v}_{b}\right) & =V^{*}\left(A_{x_{b}}^{b}\right)=\sum_{r \in \hat{\mathbb{B}}_{b}} \sum_{o \in \mathbb{O}} l_{r, o}^{x} a_{r, o} & \text { for all } b \in \mathbb{B} \quad \text { and } \\
k_{s}\left(x_{s}, \mathbf{c}_{s}\right)=V^{*}\left(A^{s}\right)-V^{*}\left(A_{\mathbb{O}_{s} \backslash x_{s}}^{s}\right) & =\sum_{r \in \hat{\mathbb{S}}_{s}} \sum_{o \in \mathbb{O}}\left[l_{r, o}^{x^{0}} a_{r, o}-l_{r, o}^{x} a_{r, o}\right] & \text { for all } s \in \mathbb{S} .
\end{aligned}
$$

That is, Definition 2 is satisfied, and so the allocation problem is assignment representable.

(Only If) Suppose that a buyer $b$ is not decomposable. Then for any matrix $A^{b}$ with $O$ unit constituents of $b$ in its rows and $O$ objects in its columns, there exists a feasible allocation $x$ such that $u_{b}\left(x_{b}, \mathbf{v}_{b}\right) \neq V^{*}\left(A_{x_{b}}^{b}\right)$. Additionally, for any $(B O+O) \times O$ unit constituent-object assignment game $A$ in which the unit constituents of $b$ are represented by the rows of $A^{b}$, a best isomorphic matching $L^{x}$ of $x$, optimally assigns objects in $x_{b}$ to unit constituents of $b$. That is,

$$
\sum_{r \in \hat{\mathbb{B}}_{b}} \sum_{o \in \mathbb{O}} l_{r, o}^{x} a_{r, o}=\sum_{r \in \hat{\mathbb{B}}_{b}} \sum_{o \in x_{b}} l_{r, o}^{x} a_{r, o}=V^{*}\left(A_{x_{b}}^{b}\right) \neq u_{b}\left(x_{b}, \mathbf{v}_{b}\right) .
$$

Consequently, the allocation problem is not assignment representable.

Likewise, if a seller $s$ is not decomposable, then for any $O_{s} \times O_{s}$ matrix $A^{s}$ there exists a feasible allocation $x$ such that $k_{s}\left(x_{s}, \mathbf{c}_{s}\right) \neq V^{*}\left(A^{s}\right)-V^{*}\left(A_{\mathbb{O}_{s} \backslash x_{s}}^{s}\right)$. Additionally, for any $(B O+O) \times O$ unit constituent-object assignment game $A$ in which the unit constituents of $s$ are represented by the rows of $A^{s}, L^{x}$, a best isomorphic matching of $x$, optimally assigns objects in $\mathbb{O}_{s} \backslash x_{s}$ to unit constituents of $s$. That is,

$\sum_{r \in \hat{\mathbb{S}}_{s}} \sum_{o \in \mathbb{O}} l_{r, o}^{x^{0}} a_{r, o}-l_{r, o}^{x} a_{r, o}=\sum_{r \in \hat{\mathbb{S}}_{s}} \sum_{o \in \mathbb{O}_{s}} l_{r, o}^{x^{0}} a_{r, o}-\sum_{r \in \hat{\mathbb{S}}_{s}} \sum_{o \in \mathbb{O}_{s} \backslash x_{s}} l_{r, o}^{x} a_{r, o}=V^{*}\left(A^{s}\right)-V^{*}\left(A_{\mathbb{O}_{s} \backslash x_{s}}^{b}\right) \neq k_{s}\left(x_{s}, \mathbf{c}_{s}\right)$.

Thus, the allocation problem is not assignment representable. 
Proof of Theorem 2: (Buyers) Let $b$ be a buyer whose utility function exhibits SDD. Then, there exists a partition $\left\{\mathbb{O}_{m}\right\}_{m=1}^{M}$ of $\mathbb{O}$ such that $\mathbb{O}_{m}=\left\{o_{(1)}\left(\mathbb{O}_{m}\right), o_{(2)}\left(\mathbb{O}_{m}\right), \ldots, o_{\left(O_{m}\right)}\left(\mathbb{O}_{m}\right)\right\}$ for all $m \in$ $\{1, \ldots, M\}$. For any package $x \in \mathbb{P}$, recall that $x_{m}:=x \cap \mathbb{O}_{m}$ and let $v_{(i)}\left(x_{m}\right):=u_{b}\left(\left\{o_{(i)}\left(x_{m}\right)\right\}, \mathbf{v}_{b}\right)$ be the $i$-th highest stand-alone utility among objects in $x_{m}$. For all $m \in\{1, \ldots, M\}$, define

$$
A^{m}:=\left(\begin{array}{cccc}
v_{(1)}\left(\mathbb{O}_{m}\right) & v_{(2)}\left(\mathbb{O}_{m}\right) & \ldots & v_{\left(O_{m}\right)}\left(\mathbb{O}_{m}\right) \\
\max \left\{v_{(1)}\left(\mathbb{O}_{m}\right)-\delta_{m, 2}, 0\right\} & \max \left\{v_{(2)}\left(\mathbb{O}_{m}\right)-\delta_{m, 2}, 0\right\} & \ldots & \max \left\{v_{\left(O_{m}\right)}\left(\mathbb{O}_{m}\right)-\delta_{m, 2}, 0\right\} \\
\vdots & \vdots & \ddots & \vdots \\
\max \left\{v_{(1)}\left(\mathbb{O}_{m}\right)-\delta_{m, O}, 0\right\} & \max \left\{v_{(2)}\left(\mathbb{O}_{m}\right)-\delta_{m, O}, 0\right\} & \ldots & \max \left\{v_{\left(O_{m}\right)}\left(\mathbb{O}_{m}\right)-\delta_{m, O}, 0\right\}
\end{array}\right)
$$

and let

$$
A:=\left(\begin{array}{cccc}
A^{m_{1}} & \mathbf{0} & \cdots & \mathbf{0} \\
\mathbf{0} & A^{m_{2}} & \cdots & \mathbf{0} \\
\vdots & \vdots & \ddots & \vdots \\
\mathbf{0} & \mathbf{0} & \cdots & A^{m_{M}}
\end{array}\right)
$$

We prove that $b$ is decomposable by showing that $A$ constitutes a decomposition of $b$. Because all other elements are zeroes, for any $x \in \mathbb{P}$, the value of $A_{x}$ (which as may be recalled is the submatrix of $A$ containing exclusively its columns related to objects in $x$ ) is maximized by separately maximizing the values of all submatrices $A_{x_{m}}^{m}$. That is,

$$
V^{*}\left(A_{x}\right)=\sum_{m=1}^{M} V^{*}\left(A_{x_{m}}^{m}\right)
$$

In order to complete the proof, it remains to show that

$$
V^{*}\left(A_{x_{m}}^{m}\right)=\sum_{i=1}^{\left|x_{m}\right|} \max \left\{u_{b}\left(\left\{o_{(i)}\left(x_{m}\right)\right\}, \mathbf{v}_{b}\right)-\delta_{m, i}, 0\right\} \quad \text { for all } m \in\{1, \ldots, M\} \text { and all } x \in \mathbb{P} \text {. }
$$

For some $m \in\{1, \ldots, M\}$ and some $x \in \mathbb{P}$, consider

$$
A_{x_{m}}^{m}=\left(\begin{array}{cccc}
v_{(1)}\left(x_{m}\right) & v_{(2)}\left(x_{m}\right) & \ldots & v_{\left(\left|x_{m}\right|\right)}\left(x_{m}\right) \\
\max \left\{v_{(1)}\left(x_{m}\right)-\delta_{m, 2}, 0\right\} & \max \left\{v_{(2)}\left(x_{m}\right)-\delta_{m, 2}, 0\right\} & \ldots & \max \left\{v_{\left(\left|x_{m}\right|\right)}\left(x_{m}\right)-\delta_{m, 2}, 0\right\} \\
\vdots & \vdots & \ddots & \vdots \\
\max \left\{v_{(1)}\left(x_{m}\right)-\delta_{m,\left|x_{m}\right|}, 0\right\} & \max \left\{v_{(2)}\left(x_{m}\right)-\delta_{m,\left|x_{m}\right|}, 0\right\} & \ldots & \max \left\{v_{\left(\left|x_{m}\right|\right)}\left(x_{m}\right)-\delta_{m,\left|x_{m}\right|}, 0\right\} \\
\vdots & \vdots & \ddots & \vdots \\
\max \left\{v_{(1)}\left(x_{m}\right)-\delta_{m, O_{m}}, 0\right\} & \max \left\{v_{(2)}\left(x_{m}\right)-\delta_{m, O_{m}}, 0\right\} & \ldots & \max \left\{v_{\left(\left|x_{m}\right|\right)}\left(x_{m}\right)-\delta_{m, O_{m}}, 0\right\}
\end{array}\right)
$$

Because the discount parameters are nondecreasing $\left(\delta_{m, i} \leq \delta_{m, j}\right.$ for $\left.i<j\right)$, the last $O-\left|x_{m}\right|$ rows of $A_{x_{m}}$ are optimally unmatched. Additionally, the max function implies that, for $i=1, \ldots,\left|x_{m}\right|$, the $i$-th row of $A_{x_{m}}^{m}$ is optimally matched to the $i$-th column. In this way, the highest value is matched to the lowest discount, then the second-highest value with the second-lowest discount, 
and so on as long as the next value is greater than or equal to the next discount. It is easy to see that this is optimal once one notices that the problem is isomorphic to the problem of a Walrasian auctioneer facing unit demand buyers with values $v_{(1)}\left(x_{m}\right), \ldots, v_{\left(\left|x_{m}\right|\right)}\left(x_{m}\right)$ and unit supply sellers with costs $\delta_{m, 1}, \ldots, \delta_{m, O_{m}}$. It follows that

$$
\left.V^{*}\left(A_{x_{m}}^{m}\right)=\sum_{i=1}^{\left|x_{m}\right|} \max \left\{v_{(i)}\left(x_{m}\right)-\delta_{m, i}, 0\right\}=\sum_{i=1}^{\left|x_{m}\right|} \max \left\{u_{b}\left(\left\{o_{(i)}\left(x_{m}\right)\right\}, \mathbf{v}_{b}\right)-\delta_{m, i}, 0\right\}\right) .
$$

(Sellers) Let $s$ be a seller whose cost function exhibits SDD. Then there exists a partition $\left\{\mathbb{O}_{m, s}\right\}_{m=1}^{M}$ of $\mathbb{O}_{s}$ such that $\mathbb{O}_{m, s}=\left\{o_{(1)}\left(\mathbb{O}_{m, s}\right), o_{(2)}\left(\mathbb{O}_{m, s}\right), \ldots, o_{\left(O_{m, s}\right)}\left(\mathbb{O}_{m, s}\right)\right\}$ for all $m \in\{1, \ldots, M\}$. For any package $x \in \mathbb{P}_{s}$, recall that $x_{m}:=x \cap \mathbb{O}_{m, s}$ and let $k_{(i)}\left(x_{m}\right):=k_{s}\left(\left\{o_{(i)}\left(x_{m}\right)\right\}\right.$, $\left.\mathbf{c}_{s}\right)$ be the $i$-th lowest stand-alone cost among objects in $x_{m}$. For all $m \in\{1, \ldots, M\}$, define

$$
A^{m}:=\left(\begin{array}{cccc}
k_{(1)}\left(\mathbb{O}_{m}\right) & k_{(2)}\left(\mathbb{O}_{m}\right) & \ldots & k_{\left(O_{m}\right)}\left(\mathbb{O}_{m}\right) \\
k_{(1)}\left(\mathbb{O}_{m}\right)+\delta_{m, 2} & k_{(2)}\left(\mathbb{O}_{m}\right)+\delta_{m, 2} & \ldots & k_{\left(O_{m}\right)}\left(\mathbb{O}_{m}\right)-\delta_{m, 2} \\
\vdots & \vdots & \ddots & \vdots \\
k_{(1)}\left(\mathbb{O}_{m}\right)-\delta_{m, O} & k_{(2)}\left(\mathbb{O}_{m}\right)-\delta_{m, O} & \ldots & k_{\left(O_{m}\right)}\left(\mathbb{O}_{m}\right)-\delta_{m, O}
\end{array}\right)
$$

and let

$$
A:=\left(\begin{array}{cccc}
A^{m_{1}} & \mathbf{0} & \cdots & \mathbf{0} \\
\mathbf{0} & A^{m_{2}} & \cdots & \mathbf{0} \\
\vdots & \vdots & \ddots & \vdots \\
\mathbf{0} & \mathbf{0} & \cdots & A^{m_{M}}
\end{array}\right)
$$

For any $m \in\{1, \ldots, M\}$, consider

$$
A_{\mathbb{O}_{m, s}^{m} \backslash x_{m}}^{m}=\left(\begin{array}{cccc}
k_{(1)}\left(\mathbb{O}_{m} \backslash x_{m}\right) & k_{(2)}\left(\mathbb{O}_{m} \backslash x_{m}\right) & \ldots & k_{\left(O_{m}-\left|x_{m}\right|\right)}\left(\mathbb{O}_{m} \backslash x_{m}\right) \\
k_{(1)}\left(\mathbb{O}_{m} \backslash x_{m}\right)+\delta_{m, 2} & k_{(2)}\left(\mathbb{O}_{m} \backslash x_{m}\right)+\delta_{m, 2} & \ldots & k_{\left(O_{m}-\left|x_{m}\right|\right)}\left(\mathbb{O}_{m} \backslash x_{m}\right)-\delta_{m, 2} \\
\vdots & \vdots & \ddots & \vdots \\
k_{(1)}\left(\mathbb{O}_{m} \backslash x_{m}\right)+\delta_{m, O_{m, s}} & k_{(2)}\left(\mathbb{O}_{m} \backslash x_{m}\right)+\delta_{m, O_{m, s}} & \ldots & k_{\left(O_{m}-\left|x_{m}\right|\right)}\left(\mathbb{O}_{m} \backslash x_{m}\right)+\delta_{m, O_{m, s}}
\end{array}\right),
$$

the matrix formed by removing all columns related to an object in $x_{m}$ from $A^{m}$. Because the discount parameters are nondecreasing $\left(\delta_{m, i} \leq \delta_{m, j}\right.$ for $\left.i<j\right)$, the first $\left|x_{m}\right|$ rows of $A_{\mathbb{O}_{m, s}^{m} \backslash x_{m}}$ optimally remain unmatched. Any assignment that matches each of the remaining $O_{m, s}-\left|x_{m}\right|$ rows to one of the $O_{m, s}-\left|x_{m}\right|$ columns produces the same value and is therefore optimal. It follows that

$$
V^{*}\left(A_{\mathbb{O}_{m, s} \backslash x_{m}}^{m}\right)=\sum_{i=1}^{O_{m}-\left|x_{m}\right|} k_{(i)\left(\mathbb{O}_{m} \backslash x_{m}\right)}+\sum_{i=\left|x_{m}+1\right|}^{O_{m}} \delta_{m, i} \quad \text { for all } m \in\{1, \ldots, M\} \text { and all } x \in \mathbb{P}_{s} .
$$


Because all other elements are zeroes, for any $x \in \mathbb{P}_{s}$, the value of $A_{\mathbb{O}_{s} \backslash x}$ is maximized by separately maximizing the values of all submatrices $A_{\mathbb{O}_{m, s} \backslash x_{m}}^{m}$. That is,

$$
V^{*}\left(A_{\mathbb{O}_{s} \backslash x}\right)=\sum_{m=1}^{M} V^{*}\left(A_{\mathbb{O}_{m, s} \backslash x_{m}}^{m}\right)=\sum_{m=1}^{M}\left[\sum_{i=1}^{O_{m}-\left|x_{m}\right|} k_{(i)\left(\mathbb{O}_{m} \backslash x_{m}\right)}+\sum_{i=\left|x_{m}\right|+1}^{O_{m}} \delta_{m, i}\right] \text { for all } x \in \mathbb{P}_{s} .
$$

It follows that, for all $x \in \mathbb{P}_{s}$,

$$
\begin{aligned}
V^{*}(A)-V^{*}\left(A_{\mathbb{O}_{s} \backslash x}\right) & =\sum_{m=1}^{M}\left[\sum_{i=1}^{O_{m}} k_{(i)\left(\mathbb{O}_{m}\right)}+\sum_{i=1}^{O_{m}} \delta_{m, i}-\sum_{i=1}^{O_{m}-\left|x_{m}\right|} k_{(i)\left(\mathbb{O}_{m} \backslash x_{m}\right)}-\sum_{i=\left|x_{m}\right|+1}^{O_{m}} \delta_{m, i}\right] \\
& =\sum_{m=1}^{M} \sum_{i=1}^{\left|x_{m}\right|}\left[k_{(i)}\left(x_{m}\right)+\delta_{m, i}\right] \\
& =\sum_{m=1}^{M} \sum_{i=1}^{\left|x_{m}\right|}\left[k_{s}\left(\left\{o_{(i)}\left(x_{m}\right)\right\}, \mathbf{c}_{s}\right)+\delta_{m, i}\right] \\
& =k_{s}\left(x, \mathbf{c}_{s}\right) .
\end{aligned}
$$

Proof of Part (ii) of Proposition 3: Suppose an agent is decomposable. Then given the agent's type, there exists a decomposition $A$. For any package $x \in \mathbb{P}$ with nonempty, disjoint subsets $y$ and $z$, part (iii) of Lemma 5 implies that,

$$
V^{*}(\tilde{A})-V^{*}\left(\tilde{A}_{.,-y}\right)+V^{*}(\tilde{A})-V^{*}\left(\tilde{A}_{.,-z}\right) \leq V^{*}(\tilde{A})-V^{*}\left(\tilde{A}_{.,-(y \cup z)}\right),
$$

where $\tilde{A}:=A_{x}$ (the matrix containing only those columns of $A$ that correspond to an object in $x$ ), and that

$$
V^{*}(\tilde{\tilde{\mathrm{A}}})-V^{*}\left(\tilde{\tilde{\mathrm{A}}}_{.,-y}\right)+V^{*}(\tilde{\tilde{\mathrm{A}}})-V^{*}\left(\tilde{\tilde{\mathrm{A}}}_{.,-z}\right) \leq V^{*}(\tilde{\tilde{\mathrm{A}}})-V^{*}\left(\tilde{\tilde{\mathrm{A}}}_{.,-(y \cup z)}\right),
$$

where $\tilde{\tilde{\mathrm{A}}}:=A_{\mathbb{O} \backslash(x \backslash(y \cup z))}$ (the matrix containing all columns of $A$ that corresponds to objects that either are in $y \cup z$ or that are not in $x$ ). If the agent is buyer $b$ with type $\mathbf{v}_{b}$, then (23) is equivalent to

$$
u_{b}\left(x, \mathbf{v}_{b}\right)-u_{b}\left(x \backslash y, \mathbf{v}_{b}\right)+u_{b}\left(x, \mathbf{v}_{b}\right)-u_{b}\left(x \backslash z, \mathbf{v}_{b}\right) \leq u_{b}\left(x, \mathbf{v}_{b}\right)-u_{b}\left(x \backslash(y \cup z), \mathbf{v}_{b}\right),
$$

which implies that buyer $b$ has substitutes payoffs. If the agent is seller $s$ with type $\mathbf{c}_{s}$, then $(24)$ is equivalent to

$$
-k_{s}\left(x \backslash(y \cup z), \mathbf{c}_{s}\right)+k_{s}\left(x \backslash z, \mathbf{c}_{s}\right)-k_{s}\left(x \backslash(y \cup z), \mathbf{c}_{s}\right)+k_{s}\left(x \backslash y, \mathbf{c}_{s}\right) \leq-k_{s}\left(x \backslash(y \cup z), \mathbf{c}_{s}\right)+k_{s}\left(x, \mathbf{c}_{s}\right),
$$

which is in turn equivalent to

$$
k_{s}\left(x, \mathbf{c}_{s}\right)-k_{s}\left(x \backslash y, \mathbf{c}_{s}\right)+k_{s}\left(x, \mathbf{c}_{s}\right)-k_{s}\left(x \backslash z, \mathbf{c}_{s}\right) \geq k_{s}\left(x, \mathbf{c}_{s}\right)-k_{s}\left(x \backslash(y \cup z), \mathbf{c}_{s}\right),
$$


which implies that seller $s$ has substitutes payoffs.

As mentioned in footnote 32 , the following lemma shows that restricting the number of unit constituents into which a buyer is decomposed to the total number of objects and restricting the number of unit constituents into which a seller is decomposed to the number of objects in his potential set is without loss of generality.

Lemma 6 A buyer $b$ who can be decomposed into any number of unit constituents can also be decomposed into $O$ unit constituents. A seller $s$ who can be decomposed into any number of unit constituents can also be decomposed into $O_{s}$ unit constituents.

Proof of Lemma 6: Consider a buyer $b$ and a set of objects $\mathbb{O}$. Let $A^{b}$ be a decomposition of $b$ containing any number of rows. $\hat{\mathbb{B}}_{b}$ is the set of unit constituents of $b$, each represented as a row of $A^{b}$. Therefore, $A^{b}$ has $\hat{B}_{b}:=\left|\hat{\mathbb{B}}_{b}\right|$ rows and $O$ columns. We need to show that for any number $\hat{B}_{b}$, there exists an $O \times O$ matrix that constitutes a decomposition of $b$. The case for a seller is analogous except that the decomposition only has $O_{s}$ columns. Hence, we only prove the lemma for a buyer.

If $\hat{B}_{b} \leq O$, then it is possible to add $O-\hat{B}_{b}$ rows of zeroes without changing the problem, so any buyer who can be decomposed into $\hat{B}_{b} \leq O$ unit constituents can also be decomposed into $O$ unit constituents.

If $\hat{B}_{b}>O$, then objects are on the short side and any optimal matching will leave at least $\hat{B}_{b}-O$ unit constituents unmatched. Let $\hat{\mathbb{B}}_{b}^{\prime} \subseteq \hat{\mathbb{B}}_{b}$ be a subset containing $\hat{B}_{b}-O$ unit constituents that are unmatched in an optimal matching of $A^{b}$. Let $\hat{A}^{b}:=A_{-\hat{\mathbb{B}}_{b}^{\prime}}^{b}$. denote the submatrix of $A^{b}$ where the rows corresponding to unit constituents in $\hat{\mathbb{B}}_{b}^{\prime}$ have been removed. Because $\hat{A}^{b}$ contains $O$ rows, the proof is complete if the following can be shown:

$$
V^{*}\left(A_{x_{b}}^{b}\right)=V^{*}\left(\hat{A}_{x_{b}}^{b}\right) \quad \text { for all } x_{b} \in \mathbb{P} .
$$

By part (v) of Lemma 5, any subset of unit constituents and any subset of objects are complements to each other in assignment game $A^{b}$. Because $\hat{\mathbb{B}}_{b}^{\prime} \subseteq \hat{\mathbb{B}}_{b}$ and $\mathbb{O} \backslash x_{b} \subseteq \mathbb{O}$ for any $x_{b} \in \mathbb{P}$, part (v) of Lemma 5 implies

$$
V^{*}\left(A^{b}\right)-V^{*}\left(\hat{A}^{b}\right)+V^{*}\left(A^{b}\right)-V^{*}\left(A_{x_{b}}^{b}\right) \geq V^{*}\left(A^{b}\right)-V^{*}\left(\hat{A}_{x_{b}}^{b}\right),
$$

which is equivalent to

$$
V^{*}\left(A^{b}\right)-V^{*}\left(\hat{A}^{b}\right) \geq V^{*}\left(A_{x_{b}}^{b}\right)-V^{*}\left(\hat{A}_{x_{b}}^{b}\right)
$$


Because the unit constituents in $\hat{\mathbb{B}}_{b}^{\prime}$ are optimally unmatched in $A^{b}, V^{*}\left(A^{b}\right)=V^{*}\left(\hat{A}^{b}\right)$ and the left side of the above inequality is equal to zero. Because removing rows always weakly reduces the value of an assignment game, the right side is also equal to zero. It follows that $V^{*}\left(A_{x_{b}}^{b}\right)=V^{*}\left(\hat{A}_{x_{b}}^{b}\right)$ for all $x_{b} \in \mathbb{P}$, and the proof is complete. 